\title{
A dynamic pricing scheme with negative prices in dockless bike sharing systems
}

\author{
J. Zhang ${ }^{1}$, M. Meng ${ }^{2}$, David, Z.W. Wang ${ }^{1 *}$ \\ 1. School of Civil and Environmental Engineering, Nanyang Technological \\ University, 50 Nanyang Avenue, Singapore 639798, Singapore \\ 2. Faculty of Business, University of Greenwich, London SE10 9LS, UK \\ *Corresponding author: Email: wangzhiwei@ntu.edu.sg
}

\begin{abstract}
To achieve bike sharing relocation ${ }^{1}$ through travellers' spontaneous behaviour, an innovative dynamic pricing strategy with negative prices is introduced in dockless bike sharing systems. In normal situation, users pay a positive price to operators for using a bike. However, when imbalanced distribution of bikes occurs in the system, users who cycle from the oversupplied area to undersupplied area will receive monetary reward from the operator, i.e., negative pricing applies. A user equilibrium dynamic traffic assignment model is developed to capture travellers' modepath choice behaviour in response to the proposed dynamic pricing strategy. Travellers can use either a single travel mode (e.g. walk, bike and bus) or combined mode (e.g. bike+bus, walk+bus, walk+bike) to complete their trips. The user equilibrium travel pattern is formulated as a variational inequality problem and then solved by a path-flow swapping algorithm. Two numerical examples are conducted to demonstrate that the proposed dynamic pricing strategy with negative price is effective in terms of attracting users as well as achieving a more balanced bike repositioning, especially when the number of bikes provided in the system is limited. Compared to free price strategy, the proposed strategy has better performance from both profit and network performance aspects.
\end{abstract}

Keywords: dynamic pricing strategy, negative price, dockless bike sharing, dynamic user equilibrium, bike relocation/repositioning

\section{Introduction}

Dockless bike sharing has recently become increasingly popular around the world. Users can unlock a public bike through scanning a smart phone application and park it at any allowed place after using it. As an emerging market, operators are trying to seize the market share by offering extremely low or even free price. For example, in Singapore, the two main operators, ofo and mobike, use a similar pricing strategy with zero deposit and low-price season ticket passes (S\$1.5 for two months and S\$5 for six months). This strategy quickly gains a large number of users and ensures market domination for both operators. Till March 2018, the number of public bikes has increased from the initial 1,000 to approximately 100,000 in Singapore within 1 year (Lim, 2018).

Due to the one-way use characteristic, there is an issue of imbalanced distribution of bikes over time and space, especially during peak period. For example, most of the

\footnotetext{
${ }^{1}$ The bike relocation mentioned in this paper is also referred as bike repositioning in the literature.
} 
users cycle from residential area to the nearest transit stations in the first-mile trip stage in the morning. Subsequently, bikes in residential area are quickly out of stock and the latter arrival users could not have the access to bikes. In this case, the level of user satisfaction may drop significantly as they cannot receive the service on time. Operators usually utilise dedicated relocation vehicles (e.g., light trucks) to move the bikes from oversupply area to undersupply area. Due to the reasons of limited resources (e.g. manpower, budget, vehicle capacity) and high relocation cost ( $\$ 3$ per bike (DeMaio, 2009)), this approach could not fundamentally solve the imbalance problem in dockless bike sharing system.

In this study, we propose a dynamic pricing strategy to guide the users' cycling behaviour so as to achieve the objective of a more balanced relocation of bikes in the system. Normally, users need to pay a positive price to the operator for the service. However, if the users park the bikes at an undersupplied area, wherein the ratio of the available bikes to the real-time demand is below certain threshold value, a radical negative price is adopted, i.e., the operator will even offer the users monetary incentives. With such a negative pricing scheme, existing bike users might adjust their routing choices while new users might be attracted from their original travel modes to use bike. Both decisions will contribute to the objective of a more balanced bike relocation. To test the performance of the proposed pricing strategy, a dynamic traffic assignment model is developed with user equilibrium principle to reflect the users' behaviour choice and estimate network flow distribution. The flow assignment results could be used for assessing the effect of the proposed pricing strategy from both profit and network performance aspects. This study has the following novel significant contributions:

(1) We introduce a dynamic pricing scheme with negative prices into bike sharing system. Indeed, to our best knowledge, this would be the first attempt to theoretically investigate the application of negative pricing scheme in an urban transportation problem.

(2) This is one of the first few studies to deal with the bike relocation problem using user-based approach in dockless bike sharing system while most of the existing solutions to bike relocation problems applied operator-based approach.

(3) A dynamic traffic assignment model and its solution algorithm are developed to capture travellers' mode-path choice behaviour in response to the proposed dynamic pricing strategy.

(4) A systematic comparison among the traditional positive price strategy, free price strategy and the proposed strategy with negative prices is analysed.

The rest of the paper is organised as follows: Section 2 briefly reviews the literature on the problem of bike sharing relocation and pricing strategy; Section 3 lays the modelling foundation as well as the definition of negative price strategy. Path travel disutility is formulated in Section 4, followed by the detailed DTA model and solution algorithm in Section 5. Section 6 gives two numerical examples to illustrate the performance of the model and the effect of the negative price strategy. Discussions and conclusions are summarised at last in Section 7.

\section{Literature review}


There are two types of approaches to solve the bike relocation problem: user-based approach and operator-based approach. The user-based approach is to guide the users to leave their bikes at specific areas to balance the bike distribution in the system. The operator-based approach is to relocate the bikes by operators using relocation vehicles (e.g. small truck). The operator-based approach is commonly used in practice, which is indeed a vehicle routing problem or pickup and delivery problem. Various models and algorithms have been developed to describe and solve the operator-based problem (Raviv et al., 2013; Chemla et al., 2013a; Dell'Amico et al., 2014; Forma et al., 2015; Erdoğan et al., 2015; Schuijbroek et al.,2017; Shui and Szeto, 2018; Szeto and Shui, 2018; Ho and Szeto, 2014; Ho and Szeto, 2017, Kadri et al., 2016, Szeto et al., 2016). Most of the existing studies dealt with the station-based bike sharing system. Nevertheless, very few researches worked on the relocation problem for the recently emerging dockless bike sharing system; meanwhile, they all applied the operator-based relocation approach. For example, Reiss and Bogenberger (2015 and 2016) used Global Positioning System (GPS) data to obtain an optimal bike distribution model and an operator-based relocation strategy was given. Pal and Zhang (2017) presented a Novel Mixed Integer Linear Program for tackling static rebalancing with single and multiple vehicles. Caggiani et al. (2018) developed a comprehensive dynamic bike relocation framework including a prediction and relocation Decision Support System.

In the literature, very few studies have been carried out to solve the bike relocation problem using user-based approaches, in either station-based bike sharing system or dockless bike sharing system. The Paris Vélib' system provides static incentives to users who return their bicycles to given stations (Laporte et al., 2015). Chemla et al. (2013b) and Pfrommer et al. (2014) proposed dynamic pricing strategies to encourage users to return bikes to empty nodes. Singla et al. (2015) extended the model of Pfrommer et al. (2014) by incorporating a crowdsourcing mechanism by providing users with alternative choices to pick or return bikes in exchange for monetary incentives. Dötterl et al. (2017) developed an event-driven agent architecture to predict the future demand with situation-aware incentives. Reiss and Bogenberger (2017) found that the user-based approach could save cost for the operator and maintain a balanced bike distribution in the system. However, existing studies have shown that the effect of user-based relocation approach is not as significant as the operator-based relocation approach in real operation (Pfrommer et al., 2014; Reiss and Bogenberger, 2017). The reason is that traditional user-based approach applies low cost pricing strategy, which may not be sufficient to induce the travellers to alter their travel behaviour. Therefore, this study introduces a new concept of negative price to achieve the objective of a more balanced bike relocation.

Generally, travellers need to pay a positive price for using a bike. Traveller is willing to pay as long as the positive utility from the received service could offset the disutility of making the payment. From the operators' point of view, the price floor is determined by the marginal cost, that is, the price can at least make a positive contribution. Normally, the marginal cost for a mature transport service (e.g. bus, metro) is greater than zero, i.e., the price of using this service is positive. The phrase of "negative price" was first introduced in the studies of good storage (Working, 1949; Wright and Williams, 1989). The price of certain good will decrease after it has been stored for some time. People still choose to store them although they might suffer loss. 
Researchers concluded that the storage leads to a negative price. Different from its initial definition of price drop after storage, negative price now has been defined as the phenomenon that customers acquire a good or service with even extra payment from sellers. Unlike below-cost price or free price, customers do not need to pay anything; instead, they receive the good or service as well as payment from seller. Some early studies also pointed out that the price of the good or service could be zero or negative from theoretical point of view (Dantzig et al., 1979; Brihaye et al., 2014). In application, the negative price strategy was firstly and mainly applied in energy field. In Germany, parts of the electricity production stemmed from wind power. As wind is an intermitting energy source, the generated power reacts strongly to the stochastic wind power infeed (Nicolosi, 2010). As the power could not be stored, when the demand is lower, the power supplier suffered from huge cost to quickly adjust the status of equipment to reduce the power generation. Until 2008, power supplier started to pay negative price to customers who consume the power when the power generation exceeds the demand. It has been verified that negative price strategy is a valid method to reduce power supplier's loss (Brandstätt et al., 2011; Simon, 2016). Another application of negative price is in internet field. Microsoft launched a Bing Cashback programme in 2008 that allowed advertisers to bid for search advertisements by offering a percentage of sales back to users (Fried, 2010). However, this programme was terminated in 2010 as the contributions from users do not achieve the desired range of vision. In this study, we investigate the feasibility of negative price concept in dockless bike sharing system. Facing unbalanced distribution in the system, operators offer rewards to the users who cycle from oversupplied area to undersupplied area. This strategy could achieve bike relocation as well as operation cost reduction, and thus bring a positive contribution.

When the negative pricing strategy is introduced in the dockless bike sharing system, it will significantly affect customers' travel choice behaviour. Dynamic traffic assignment (DTA) model is one of the key methods to support the evaluation of different pricing strategies in a network context. DTA was first proposed by Merchant and Nemhauser (1976) in considering time varying flows. Various DTA methods (e.g. analytical models, simulation models, and cell models) have been developed and applied in transportation analysis (Lo and Szeto, 2004; Szeto and Lo, 2006; Wang and Du, 2016; Liu and Geroliminis, 2017; Liu and Szeto, 2019; Zhang and Liu 2019). One major application of DTA is the evaluation of dynamic road pricing strategies (Lu et al., 2008; Zheng et al., 2012; Zhang et al., 2013). Some researchers have tested the effect of different pricing strategies in taxi market (Ciari et al., 2015; Qian et al., 2017; Long et al., 2017). Recently, Li et al. (2018) examined the different pricing strategies in free-floating car-sharing scheme and found that rental price has significant implications for travel demand management. Xu et al., (2018) also pointed out the demand for car-sharing service is elastic with respect to the trip price. These studies all followed the traditional pricing strategy with positive price at any time. The application of DTA model in bike sharing system is mainly in the area of fleet allocation. Usually, a time-space network is constructed to describe the dynamic bike-sharing demand (Ghoshe t al, 2017; Lu, 2016; Regue and Recker, 2014; Shui and Szeto, 2018; Yan et al, 2017; Caggiani et al., 2018), while the fleet allocation is formulated as mixed integer programme. The usage of DTA model to discuss the influence of pricing strategy has 
not been fully discussed in the literature. This study would find out whether the proposed pricing strategy is feasible and effective in dockless bike sharing system.

\section{Basic considerations}

\subsection{Assumptions}

Some assumptions are made prior to the modelling as follows:

A1. The study time horizon $[0, \mathrm{~T}]$ is discretised into a finite set of equal duration time intervals, i.e., $\{1,2,3, \quad, \bar{T}\}$. Let $\Delta$ be the duration of each time interval $\Delta \cdot \bar{T}=T$.

A2. The study focuses on first/last mile trip, where three common travel modes are considered including walk, bike and bus. Travellers all have the access to dockless public bikes and do not own their private bikes.

A3. Travellers may use one or up to three modes in a trip. In other words, maximum two transfers are allowed.

A4. The heterogeneity of traveller is considered in terms of a discrete set of value of time (VOT).

A5. Bus runs on dedicated bus lanes so that bus travel time is fixed according to the timetable and is not affected by other modes.

A6. Bidirectional flow on walk and bike links are considered in a separate way. There is no mixed-traffic flow on all links.

A7. All travellers will check the bike availability and price before making a mode-path choice. They can reserve any available bike regardless the distance. The reservation follows first-reserve-first-use principle.

A8. There are limited number of bikes provided in the network. If there are no bikes available, travellers at this location could only choose to walk or take bus.

A9. All travellers who take the negative price reward will follow the rules to return the bikes on time.

\subsection{Notation}

Primary notation used in this study are defined in Table 1 as follows:

Table 1 Notations

\begin{tabular}{ll}
\hline Sets & Set of nodes \\
$N$ & Set of total time \\
$T$ & Set of Origin-Destination (OD) pairs \\
$W$ & Set of paths \\
$P$ & Set of links \\
$L$ & Set of links going out of node $i, A(i)=\{(i, j) \in \mathrm{A}\}$ \\
$A(i)$ & Set of incoming links of node $i, B(i)=\{(j, i) \in \mathrm{A}\}$ \\
$B(i)$ & Set of travel mode, $\{M \mid M=$ walk, bike, bus $\}$ \\
$M$ & \\
Indices & \\
\hline
\end{tabular}




\begin{tabular}{|c|c|}
\hline$w, w^{\prime}$ & OD pair, $w \in W$ \\
\hline$p, p^{\prime}, \bar{p}$ & Path $p, p^{\prime}, \bar{p} \in P$ \\
\hline$l, l^{\prime}, l_{+}, l_{-}$ & Travel link, $l, l^{\prime}, l_{+}, l_{-} \in L$ \\
\hline$i, j$ & Nodes \\
\hline$r, s$ & Origin and destination nodes of $w$ \\
\hline$r_{\text {bike }}, s_{\text {bike }}$ & Origin and destination nodes of bike stage \\
\hline$m, m^{\prime}$ & Travel mode, $m, m^{\prime} \in M$ \\
\hline$t, t^{\prime}$ & Time intervals \\
\hline$k$ & Passenger groups according to value of time \\
\hline \multicolumn{2}{|c|}{ Parameters } \\
\hline$\zeta^{k}$ & Value of time for group $k$ \\
\hline$\lambda$ & Comfort loss-time conversion coefficient \\
\hline$\eta_{m}$ & Comfort loss of mode $m$ per time \\
\hline$g_{\text {bike }}^{b v}$ & $\begin{array}{l}\text { Large value, to impose extremely high cost for the case with no } \\
\text { available bikes }\end{array}$ \\
\hline$g_{\text {bike }}^{\text {pos }}$ & Normal positive price of renting bike \\
\hline$Q_{\text {bike }}$ & Supply of bikes \\
\hline$\varepsilon$ & Tolerance level \\
\hline \multicolumn{2}{|l|}{ Variables } \\
\hline$P U^{w, p, k}(t)$ & Disutility of $w$ via $p$ for group $k$ at time $t$ \\
\hline$L U^{l, k}(t)$ & Disutility of group $k$ on link $l$ at time $t$ \\
\hline$\delta^{w, p, l, t^{\prime}}(t)$ & $\begin{array}{l}\text { Indicator variable: traveller of } w \text { depart at } t \text { via } p \text { and entry link } l \text { at } t^{\prime} \text {, } \\
\text { equal to } 1 \text {; otherwise } 0\end{array}$ \\
\hline$T_{m}^{l, k}(t)$ & Time cost of mode $m$ for group $k$ on link $l$ at time $t$ \\
\hline$G_{m}^{l, k}(t)$ & Monetary cost of mode $m$ for group $k$ on link $l$ at time $t$ \\
\hline$C_{m}^{l, k}(t)$ & Comfort cost of mode $m$ for group $k$ on link $l$ at time $t$ \\
\hline$t_{0, m}^{l}$ & Free flow time of mode $m$ on link $l$ \\
\hline$x_{m}^{l}(t)$ & Flow of mode $m$ on link $l$ at time $t$ \\
\hline$\Phi_{t h, 1}^{i}(t)$ & $\begin{array}{l}\text { threshold value of bike supply at node } i \text { at time } \mathrm{t} \text { below which } \\
\text { undersupply is defined }\end{array}$ \\
\hline$\Phi_{t h, 2}^{i}(t)$ & $\begin{array}{l}\text { threshold value of bike supply at node } i \text { at time } \mathrm{t} \text { above which } \\
\text { oversupply is defined }\end{array}$ \\
\hline$\Phi_{b i k e}^{i}(t)$ & value of bike supply at node $i$ at time $\mathrm{t}$ \\
\hline$f^{w, p, k}(t)$ & Flow for group $k$ of $w$ via $p$ at time $t$ \\
\hline$\Gamma^{w, p, k *}(t)$ & Minimum disutility for group $k$ of $w$ via $p$ at time $t$ \\
\hline$u^{w, p, l}(t)$ & Inflow of $w$ via $p$ on link $l$ at time $t$ \\
\hline$v^{w, p, l}(t)$ & Outflow of $w$ via $p$ on link $l$ at time $t$ \\
\hline$f^{w}(t)$ & Flow of $w$ departing at time $t$ \\
\hline$e^{w}(t)$ & Flow of $w$ arriving at time $t$ \\
\hline
\end{tabular}


$E_{m}^{w, p, k}(t) \quad$ Cumulative flow of mode $m$ for group $k$ of $w$ via $p$ by time $t$

$\tau_{m}^{l}(t) \quad$ Travel time of mode $m$ on link $l$ depart at time $t$

\subsection{Multimodal Supernetwork}

Supernetwork is abstracted from a normal network, wherein the hierarchy of real network and the virtual connection among the network are both included. In this study, a multimodal transportation supernetwork is constructed. Firstly, the study area is divided into certain number of traffic analysis zones (TAZs), where each TAZ is connected with real road links. As users can park the bikes at any location in the dockless bike sharing system, the size of one TAZ is usually selected to encapsulate one or several buildings within the field of human vision. Secondly, supplementary virtual links are added to the real transportation network to support the full description of travel behaviour, such as access link, transfer link as well as the weight on each link. Accordingly, an illustrative transportation network as in Figure 1 could be expanded and represented by a supernetwork as shown in Figure 2, wherein the solid lines represent the running links and the virtual links represent the access and transfer links. The weight on each link could be various attributes, such as travel time, monetary cost, comfort loss.

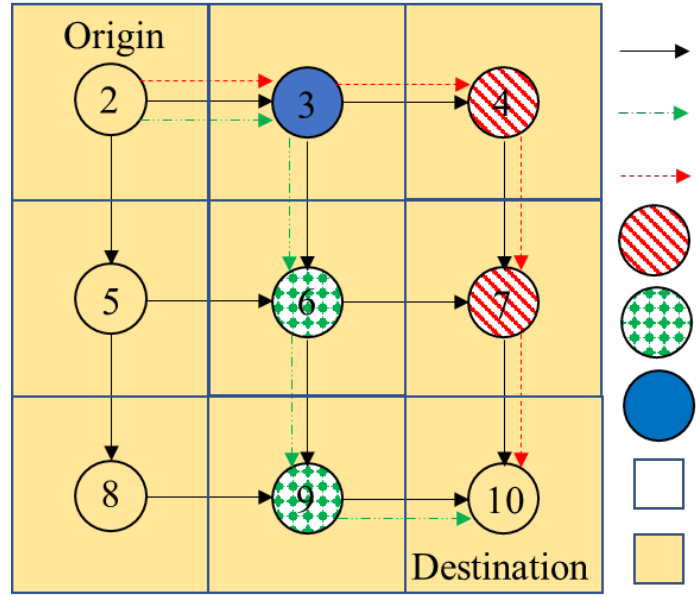

Figure 1 Study Area

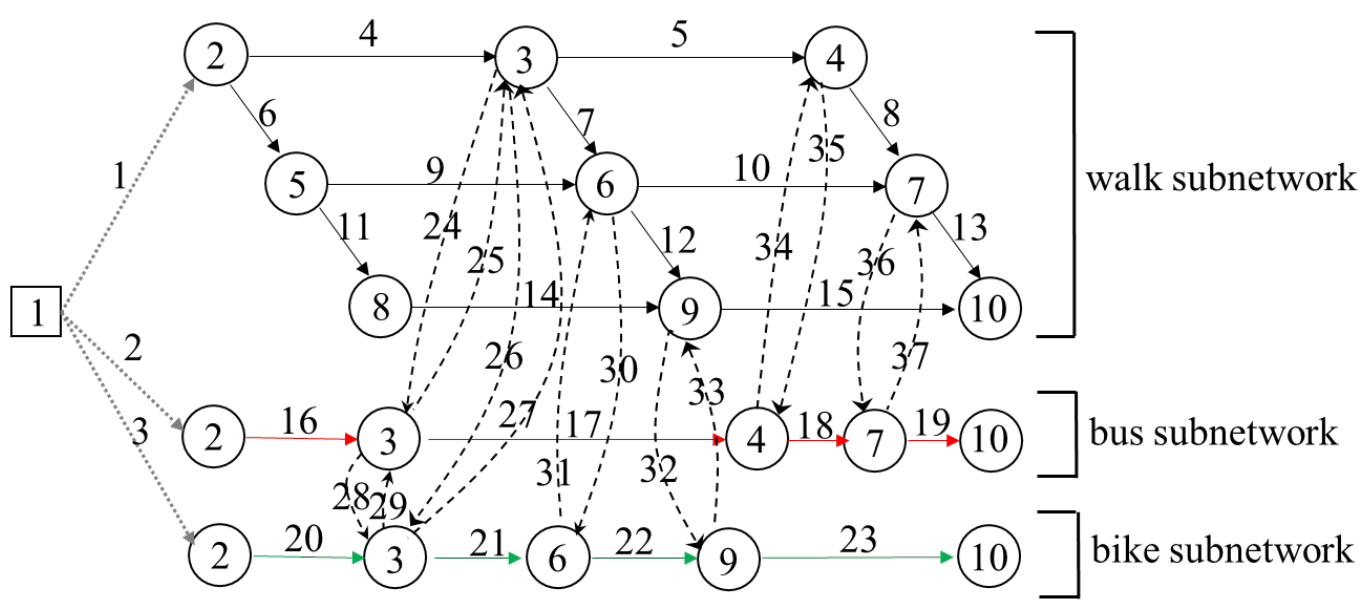

Figure 2 Supernetwork 
A path in supernetwork is called superpath. Not all superpaths can describe actual travel routes. For example, travellers will not have unlimited transfers in one trip. Based on the assumptions in this study, a superpath is defined to be feasible only if it has maximum two transfers and no two continuous transfer links between one OD pair is allowed. For example, in Figure 2, path 1-4-24-17-18-19 is a feasible superpath between OD pair $(1,10)$ while path 1-4-26-29-17-18-19 is not a feasible superpath between OD pair $(1,10)$ as there are two continuous transfer links 26 and 29.

\subsection{Negative price scheme}

As mentioned above, negative price strategy in this study means that user could get monetary reward from operator. The negative price scheme is only applicable when users cycle from oversupply area to undersupply area so that the bike relocation is achieved in the system. Three statuses are defined for each TAZ in the study area, i.e., undersupply, normal and oversupply:

$$
\operatorname{status}(i, t)=\left\{\begin{array}{l}
\text { undersupply } \quad \text {, if } \Phi_{b i k e}^{i}(t) \leq \Phi_{t h, 1}^{i}(t) \\
\text { oversupply, if } \Phi_{b i k e}^{i}(t)>\Phi_{t h, 2}^{i}(t) \\
\text { normal, otherwise }
\end{array}, \forall \Phi_{t h, 1}^{i}(t)<\Phi_{t h, 2}^{i}(t), i \in N(1)\right.
$$

Therefore, the dynamic pricing scheme in this study is proposed as: user will pay a positive price if the status of the origin TAZ or destination TAZ is normal; Negative price applies when the status of the origin TAZ is oversupply and the destination TAZ is undersupply. To illustrate the performance of the proposed strategy, two common pricing strategies are also considered as the comparison benchmarks: traditional fixed pricing strategy where the price is a constant positive value, and free pricing strategy. For simplification purpose, we abbreviate the proposed negative price strategy as NP, traditional positive price strategy as PP, and free price strategy as FP in the rest of the paper.

The negative price value is assumed to be dependent on the level of undersupply or scarcity degree of bikes at the destination nodes. Hereby, we propose a simple linear relationship between the negative price and the level of undersupply of bikes. The structure of the pricing scheme can be schematically depicted in Figure 3. Basically, if the bike trip starts from an oversupplied location to an undersupplied one, the negative price will apply; otherwise, the normal positive price will be adopted. Bounded by a maximum value, the negative price is set to be linear with respect to the level of undersupply. 


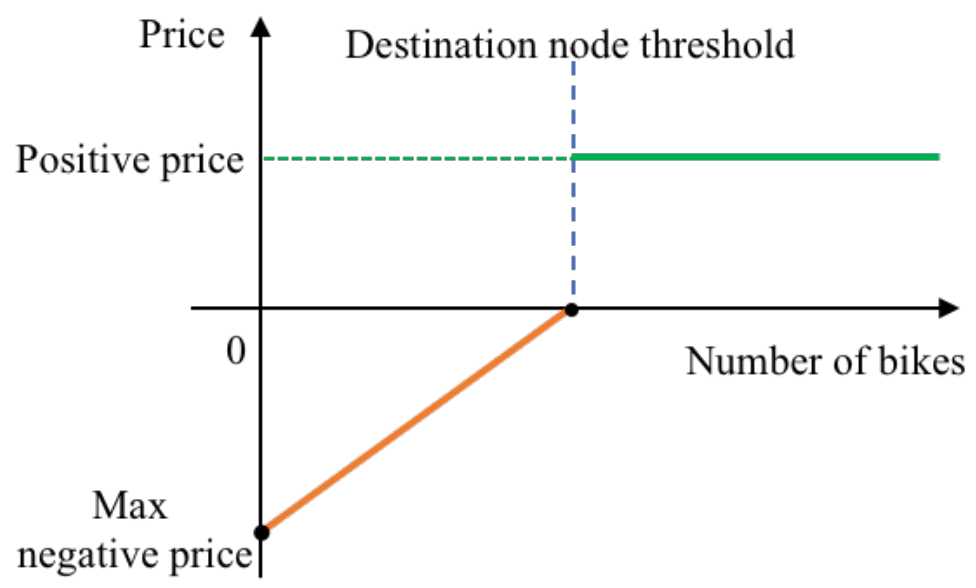

Figure 3 Negative price scheme

Based on the above analysis, the dynamic pricing scheme with negative price can be proposed as follows:

$$
G_{\text {bike }}(t)=\left\{\begin{array}{l}
g_{\text {bike }}^{\text {neg }}(t) \cdot\left[\frac{\Phi_{\text {bike }}^{s_{\text {bike }}}(t)-\Phi_{t h, 1}^{s_{\text {bike }}}(t)}{\Phi_{\text {th. }}^{s_{\text {bike }}}(t)}\right], \forall\left\{\begin{array}{l}
\Phi_{\text {bike }}^{r_{\text {bike }}}(t)>\Phi_{\text {thit. }}^{r_{\text {bike }}}(t) \\
\Phi_{\text {bike }}^{s_{\text {bike }}}(t) \leq \Phi_{\text {th }, 1}^{s_{\text {bike }}}(t)
\end{array}\right. \\
g_{\text {bike }}^{\text {pos }}, \text { otherwise }
\end{array}\right.
$$

Here, when users cycle bikes from an oversupplied area to an undersupplied area, a negative price applies, wherein the value of negative price is set to be determined by the level of undersupply at the destination TAZ (i.e., $\frac{\Phi_{\text {bike }}^{s_{\text {bike }}}(t)-\Phi_{t h, 1}^{s_{\text {bike }}}(t)}{\Phi_{\text {th. }}^{s_{\text {bike }}}(t)}$ ) and the predefined maximum negative price $g_{\text {bike }}^{\text {neg }}(t)$. That is, to determine the negative price, it is required to know the supply of bikes $\Phi_{\text {bike }}^{s_{b i k e}}(t)$, as well as the defined threshold value of undersupply $\Phi_{t h, 1}^{s_{b i k e}}(t)$. It is noted that, the real-time information of bike supply can be easily obtained in practice from the GPS data. On the other hand, the key is to determine how to set the threshold value for undersupply. It is not difficult to understand that the threshold value for undersupply is highly related to the bike travel demand. In practice, the bike demands at different locations could be estimated by adopting prediction methods, such as the Auto Regressive Conditional Heteroscedasticity (ARCH) model, based on the historical and real-time data. In the proposed negative pricing scheme in this study, we set the threshold value for undersupply by assuming a simple linear relationship with the predicted demand value of $Q(i, t)$, i.e., $\Phi_{t h}^{i}(t)=Q(i, t)+\phi$. Herein, the parameter $\phi$ could be determined based on the specific objectives, e.g., attracting more users or maximising fare revenue. In practice, other than applying rigorous optimisation modeling approach, one can obtain the optimal value of the parameter $\phi$ by conducting a simple sensitivity analysis approach.

Proposition 1 With a sufficient number of bikes in market, NP will reduce to PP. 
Proof. From Eq. (2), it is known that negative price occurs when the relationship between bike supply and the threshold values at the origin and destination area satisfies $\Phi_{\text {bike }}^{r_{\text {ikike }}}(t)>\Phi_{\text {th,2 }}^{r_{\text {bike }}}(t), \Phi_{\text {bike }}^{s_{\text {bike }}}(t) \leq \Phi_{t h, 1}^{s_{\text {bike }}}(t)$. The threshold values $\Phi_{\text {th, }}^{r_{\text {bike }}}(t)$ and $\Phi_{\text {th,1 }}^{s_{\text {bike }}}(t)$ are highly relevant to dynamic bike travel demand at certain location. As the bike supply level in the market goes up, $\Phi_{\text {bike }}^{s_{\text {bike }}}(t)$ will increase and therefore the probability of fulfilling negative price condition $\Phi_{\text {bike }}^{s_{\text {bike }}}(t) \leq \Phi_{t h, 1}^{s_{\text {bike }}}(t)$ will reduce. If the bike supply in the market is sufficiently large, the negative price conditions would not be satisfied, i.e., the proposed NP scheme defined in (2) will reduce to PP.

Essentially, two types of behaviour changes might happen if NP is adopted: One is the change of travel mode, that is, the non-cycling travel mode users would use bikes and end the trips at the undersupply TAZ. The other one is the change of travel path, that is, the existing bike users would use another path and end the trip at the undersupply TAZ. For example, in Figure 4, node 1 is the origin node and node 3 is the destination node. For the travellers who usually take bus from node 1 to node 3 , after NP is adopted, they might choose to cycle from node 1 to node 2 and then take bus from node 2 to node 3 , as the reward from negative price scheme might offset the incurred extra travel disutility. Similarly, for an existing bike user from node 1 to node 3 , he/she might park the bike at node 2 and then walk or take bus from node 2 to node 3 , if reward from negative price could offset the added disutility. Both of these travel behaviour changes mentioned above can contribute to the bike relocation. In this case, operators are able to reduce the operation cost for bike relocation. The achieved real-time relocation through travellers' spontaneous travel behaviour could also improve the level of service, and thus bring new users and increase revenue.

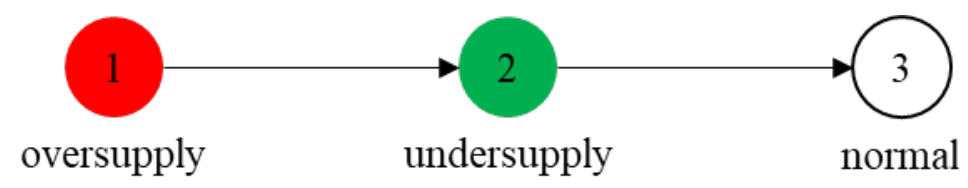

Figure 4 A super path with three nodes

Proposition 2 Under NP, for the first type of behaviour change, i.e., switching from non-cycling mode to cycling mode, one bike will at least serve two users. If the maximum NP value is set to be not greater than PP fare, non-negative fare revenue could be obtained.

Proof. Under NP, if a traveller changes travel mode, he/she will relocate a bike from an oversupplied area to an undersupplied area where demand is larger than supply. Then, another user will be able to ride this bike to other places. In this case, this bike will be used by at least two travellers. If the traveller selects his/her original non-cycling travel mode, the bike sharing service operator achieve zero fare revenue. If mode switching occurs, from Eq. (2), the fare revenue for the first trip is subject to NP scheme $\left(g_{\text {bike }}^{\text {neg }}(t) \cdot\left[\Phi_{t h, 1}^{s_{\text {bike }}}(t)-\Phi_{\text {bike }}^{s_{\text {bike }}}(t)\right] / \Phi_{\text {th.1 }}^{s_{\text {bike }}}(t)\right)$, while the second trip follows PP $\left(g_{\text {bike }}^{\text {pos }}\right)$. In this case, the total direct fare revenue can be rewritten as $-g_{\text {bike }}^{\text {neg }}(t) \cdot \Phi_{\text {bike }}^{s_{\text {bike }}}(t) / \Phi_{\text {th. } 1}^{s_{\text {bike }}}(t)+g_{\text {bike }}^{\text {pos }}+g_{\text {bike }}^{\text {neg }}(t)$. If $-g_{\text {bike, } \max }^{\text {neg }} \leq g_{\text {bike }}^{\text {pos }}$, the fare revenue must be positive. 
Proposition 3 Under NP, for the second type of behaviour change, i.e., the travel path adjustment, more users would be served and positive fare revenue will be obtained with nevertheless smaller amount of fare revenue.

Proof. Under NP, if a traveller decides to change path, he/she must switch from a path with positive price to a path with negative price. The original fare revenue is $g_{b i k e}^{\text {pos }}$. The new trip after changing would apply negative pricing, which means that the new destination is undersupplied. In this case, the bike will be used by another user with positive price, and more users would be served as compared with PP. The new fare revenue is $g_{\text {bike }}^{\text {pos }}+g_{\text {bike }}^{\text {neg }}(t) \cdot\left[\frac{\Phi_{\text {bike }}^{s_{\text {bike }}}(t)-\Phi_{\text {th }, 1}^{s_{\text {bike }}}(t)}{\Phi_{\text {th.1 }}^{s_{\text {bike }}}(t)}\right]$. As $\Phi_{\text {bike }}^{s_{\text {bike }}}(t)<\Phi_{\text {th }, 1}^{s_{\text {bike }}}(t)$, we have $g_{\text {bike, } \max }^{\text {neg }} \leq g_{\text {bike }}^{\text {neg }}(t) \cdot\left[\Phi_{\text {bike }}^{s_{\text {bike }}}(t)-\Phi_{t h, 1}^{s_{\text {bike }}}(t)\right] / \Phi_{\text {th.1 }}^{s_{\text {bike }}}(t)<0$. If it is assumed $-g_{\text {bike, } \max }^{\text {neg }} \leq g_{\text {bike }}^{\text {pos }}$, fare revenue would be positive, but it is still less than original fare revenue.

A set of illustrative examples with three scenarios is given to demonstrate the effectiveness of NP as compared to traditional PP scheme and FP scheme. Assume the original travel modes in three scenarios are all under PP, where traveller travels from node 1 to node 3 passing node 2 as shown in Figure 3. Node 1 is in the status of oversupply, while node 2 undersupply and node 3 normal. Two links share the same distance in three scenarios. Three strategies are given for three scenarios: FP(1-2-3) represent the scenario that the whole network is under FP, i.e., with free charge; FP (12 ) is that link connecting node 1 and node 2 is under FP while the other link is under PP; NP (1-2) is that link connecting node 1 and node 2 is under NP while the rest link is still under PP. The highly possible travel decisions under different pricing strategies are listed in Table 2. From Table 2, it could be concluded that FP (1-2-3) cannot achieve bike relocation while FP (1-2) and NP (1-2) are able to do so. The behaviour adjustment in Scenario A belongs to the second type change, i.e., path changing, while Scenarios $\mathrm{B}$ and $\mathrm{C}$ apply mode switching. In Scenario $\mathrm{B}$, the original travel mode is walking from node 1 to node 3 . When FP is offered from node 1 to node 2 , travellers are not likely to choose to use bike as they need to take extra effort (disutility) to cycle. However, if extra reward is given, travellers may choose cycling from node 1 to node 2 as the reward might offset their added disutility from using bike. Therefore, NP (1-2) is more effective than FP (1-2-3) and FP (1-2) in terms of attracting travellers to cycle and thus achieving a more balanced relocation. In reality, NP and FP could directly attract large number of users in a short time and indirectly reduce the advertisement cost. With the huge number of users, ancillary revenue other than fare revenue could be generated. Unlike belowcost price, free price and negative price have highly likelihood to let users overvalue the service because humans naturally fear of loss. Free price and negative price will not lead to any loss while below cost price still have a risk of loss.

Table 2 Highly possible travel behaviour under different pricing strategies

\begin{tabular}{clrll}
\hline Scenario & PP(original) & FP (1-2-3) & \multicolumn{1}{c}{ FP (1-2) } & \multicolumn{1}{c}{ NP (1-2) } \\
\hline \multirow{2}{*}{ A } & bike:1-2-3 & bike: $1-2-3$ & bike:1-2-3 & bike:1-2-3 \\
& & & or bike:1-2 bus 2-3 & or bike:1-2 bus 2-3
\end{tabular}




\begin{tabular}{cclll}
\hline & & or bike 1-2 walk 2-3 & or bike 1-2 walk 2-3 \\
B & walk:1-2-3 & walk:1-2-3 & walk:1-2-3 & walk:1-2-3 \\
& & or bike:1-2-3 & walk: & or bike:1-2 walk 2-3 \\
& & bus:1-2-3 & bus:1-2-3 & bus:1-2-3 \\
C & bus:1-2-3 & or bike:1-2-3 & & or bike:1-2 bus 2-3 \\
\hline
\end{tabular}

\section{Path travel disutility}

Based on the assumption that the travel disutility on each link is independent, the generalised path travel disutility could be calculated by summing up the corresponding link disutility on this path as follows:

$$
P U^{w, p, k}(t)=\sum_{l} \sum_{t} L U^{l, k}(t) \cdot \delta^{w, p, l, t^{\prime}, k}(t), \forall w, p, l, k, t, t^{\prime}
$$

For each link, the link travel disutility includes three components, travel time, monetary cost and comfort loss (discomfort). Travel time consists of walking time, cycling time, in-vehicle time and waiting time. Considering the weight of each component, the link travel disutility could be expressed as:

$$
L U^{l, k}(t)=\omega_{T} T^{l, k}(t)+\omega_{G} G^{l, k}(t)+\omega_{C} C^{l, k}(t), \forall l, k
$$

where $\omega_{T}, \omega_{G}$ and $\omega_{C}$ are weight parameters, $\omega_{T}+\omega_{G}+\omega_{C}=1$.

In a multimodal network with consideration of the combined travel mode, there are three types of travel links, i.e., running links, transfer links and access links. Running links include walk links, bike links and bus links. For simplification purpose, the monetary cost on bike and bus links is moved to the connected access link.

\subsection{Walk link disutility}

Pedestrian walks on the walk link without being affected by other modes. The interaction of bi-direction pedestrian flow on walk link is considered. Travel time for walking is calculated as in Wu and Lam (2003). There is no monetary cost on walk link. Comfort loss (discomfort) on walk link is directly related to the travel time. Thus, three components in walk link disutility are calculated by:

$$
T_{\text {walk }}^{l_{+}}(t)=t_{0, \text { walk }}^{l_{+}}+A_{\text {walk }}\left[\frac{x_{\text {walk }}^{l_{+}}(t)}{x_{\text {walk }}^{l_{+}}(t)+v_{\text {walk }}^{l_{-}}(t)}\right]^{\alpha_{\text {walk }}}\left[\frac{x_{\text {walk }}^{l_{+}}(t)}{B_{\text {walk }}^{l}}\right]^{\beta_{\text {walk }}}, \forall l, l_{+}, l_{-} \in L_{\text {walk }}
$$

where $l_{+}$and $l_{-}$represent the two directions on link $l . A_{\text {walk }}, B_{\text {walk }}^{l}, \alpha_{\text {walk }}$ and $\beta_{\text {walk }}$ are parameters to be calibrated with observed data.

$$
\begin{gathered}
G_{\text {walk }}^{l}(t)=0, \forall l \in L_{\text {walk }} \\
C_{\text {walk }}^{l}(t)=\lambda \eta_{\text {walk }} T_{\text {walk }}^{l}(t), \forall l \in L_{\text {walk }}
\end{gathered}
$$

4.2 Bike link disutility 
Similarly, cyclists do not share the bike link with pedestrians or bus passengers. The interaction of bi-direction cyclist flow on bike link is also considered in the travel time calculation. The monetary cost is assumed to be dependent on the supply and demand interaction at the departure time, and not relevant with the travel time. Thus, the monetary cost on bike link can be transferred to the connected access link. As cycling is a human-powered travel mode, comfort loss on bike link is closely related to the travel time. Then, the three components in bike link disutility are given by:

$$
\begin{gathered}
T_{\text {bike }}^{l_{+}}(t)=t_{0, \text { bike }}^{l_{+}}+A_{\text {bike }}\left[\frac{x_{\text {bike }}^{l_{+}}(t)}{x_{\text {bike }}^{l_{+}}(t)+x_{\text {bike }}^{l_{-}}(t)}\right]^{\alpha_{\text {bike }}}\left[\frac{x_{\text {bike }}^{l_{+}}(t)}{B_{\text {bike }}^{l}}\right]^{\beta_{\text {bike }}}, \forall l, l_{+}, l_{-} \in L_{\text {bike }} \\
G_{\text {bike }}^{l}(t)=0, \forall l \in L_{\text {bike }} \\
C_{\text {bike }}^{l}(t)=\lambda \eta_{\text {bike }} T_{\text {bike }}^{l}(t), \forall l \in L_{\text {bike }}
\end{gathered}
$$

\subsection{Bus link disutility}

Bus service is assumed to follow strictly the running time on timetable, which is not affected by other modes. Assuming that a flat fare ticket structure applies, one can move the monetary cost on bus link to the connected access link. Besides the travel time, passengers may feel discomfort on the bus due to the crowded environment in peak period. Then, the three components in bus link disutility could be obtained as follows:

$$
\begin{gathered}
T_{b u s}^{l}(t)=t_{0, b u s}^{l}, \forall l \in L_{b u s} \\
G_{b u s}^{l}(t)=0, \forall l \in L_{b u s} \\
C_{b u s}^{l}(t)=\lambda \eta_{b u s} T_{b u s}^{l}(t)+\left[\frac{v_{b u s}^{l}(t)}{D_{b u s} Y_{b u s}^{l}}\right], \forall l \in L_{b u s}
\end{gathered}
$$

where $D_{\text {bus }}$ is bus capacity and $Y_{\text {bus }}^{l}$ is capacity of link $l$.

\subsection{Transfer link disutility}

Travel time on transfer link includes transfer walking time and transfer waiting time. Transfer walking time is approximately equal to the ratio of transfer walking distance to the average walking speed. Transfer waiting time is incurred only for transfer from walk or bike link to bus link, which is equal to half of bus headway. There is no monetary cost on transfer link. Comfort loss on transfer link is decided by the transfer walking time. The three components in transfer link disutility are expressed as follows:

$$
\begin{gathered}
T_{m, m^{\prime}}^{l}(t)=t_{m, m^{\prime}}^{l}, \forall m, m^{\prime} \in M, l \in L_{\text {transfer }} \\
G_{m, m^{\prime}}^{l}(t)=0, \forall m, m^{\prime} \in M, l \in L_{\text {transfer }} \\
C_{m, m^{\prime}}^{l}(t)=\lambda \eta_{\text {walk }} t_{m, m^{\prime}}^{l}, \forall m, m^{\prime} \in M, l \in L_{\text {transfer }}
\end{gathered}
$$

4.5 Access link disutility 
Travel time on access link is zero if the access link is connected to walk or bike link, and equal to half of the bus headway if the access link is connected to bus link. Monetary cost on access link could be bike rental fee or bus fare cost. There is no comfort loss on access link. The three components in access link disutility are expressed as follows:

$$
\begin{gathered}
T_{a e}^{l}(t)=T_{m}^{\text {wait }}(t), \forall l \in L_{a e} \\
G_{a e}^{l, k}(t)=\zeta^{k} G_{m}^{k}(t), \forall l \in L_{a e} \\
C_{a e}^{l}(t)=0, \forall l \in L_{a e}
\end{gathered}
$$

where $T_{\text {walk }}^{\text {wait }}=T_{\text {bike }}^{\text {wait }}=0, T_{\text {bus }}^{\text {wait }}=H / 2$.

Bike rental fee follows the dynamic pricing scheme, which can be expressed as follows:

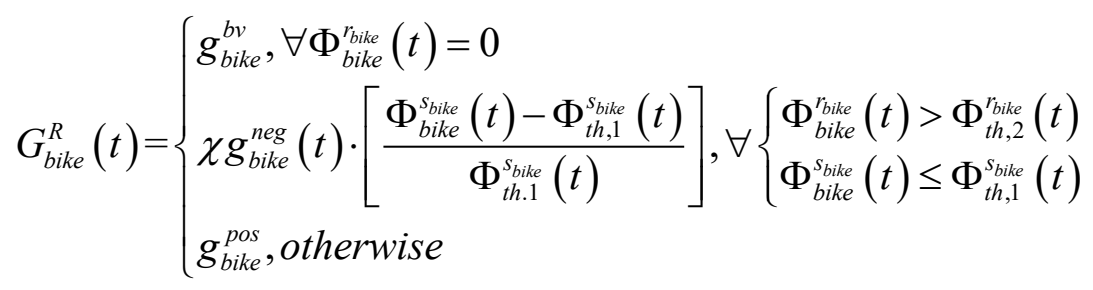

Here, when bike supply is zero, a prohibitively large value of cost for using bikes is imposed. $\chi$ is a constant coefficient to represent contrast preference from negative price. According to the theory of loss aversion, when people face the same amount of gains and losses, losses are more intolerant than gains. The disutility that comes from losses is 2.25 times of the utility from gains (Tversky and Kahneman, 1992).

\section{Model and algorithm}

\subsection{Dynamic user equilibrium principle}

According to assumption A4, we consider $k$ classes of travellers with different values of time. The dynamic user equilibrium condition is defined as: for each class $k$ and each OD pair at each time interval, the total travel disutility for all superpaths that are being used equal to the minimal total super path travel disutility. It could be formulated as:

$$
\begin{gathered}
P U^{w, p, k *}(t)-\Gamma^{w, p, k^{*}}(t) \geq 0, \quad \forall w, p, k \\
f^{w, p, k *}(t) \cdot\left[P U^{w, p, k^{*}}(t)-\Gamma^{w, p, k *}(t)\right]=0, \quad \forall w, p, k \\
f^{w, p, k}(t) \geq 0, \quad \forall w, p, k
\end{gathered}
$$

5.2 Dynamic network constraints

Five types of constraints are given according to the model requirements. First, the basic definitional constraints are expressed as:

$$
\sum_{w} \sum_{p} \sum_{k} x_{m}^{w, p, l, k}(t)=x_{m}^{l}(t), \forall w, p, l, k, m
$$




$$
\begin{gathered}
\frac{d x_{m}^{w, p, l, k}(t)}{d t}=u_{m}^{w, p, l, k}(t)-v_{m}^{w, p, l, k}(t), \forall w, p, l, k, m \\
\sum_{w} \sum_{p} \sum_{k} u_{m}^{w, p, l, k}(t)=u_{m}^{l}(t), \forall w, p, l, k, m \\
\sum_{w} \sum_{p} \sum_{k} v_{m}^{w, p, l, k}(t)=v_{m}^{l}(t), \forall w, p, l, k, m
\end{gathered}
$$

Second, at the origin and destination nodes, flow conservation constraints satisfy:

$$
\begin{gathered}
f_{m}^{w, k}(t)=\sum_{l \in A(r)} \sum_{p} u_{m}^{w, p, l, k}(t), \forall w, p, k, m \\
\sum_{l \in B(s)} \sum_{p} v_{m}^{w, p, l, k}(t)=e_{m}^{w, k}(t), \forall w, p, k, m
\end{gathered}
$$

where $f_{m}^{w, k}(t)$ denote the $m$ mode flow of class $k$ departing at origin node to destination node at time $t$, and $e_{m}^{w, k}(t)$ denote the $m$ mode flow of class $k$ arriving at destination from origin at time $t$. For all the other nodes, flow conservation constraints can be written as:

$$
\sum_{l \in A(i)} v_{m}^{w, p, l, k}(t)=\sum_{l \in B(i)} u_{m}^{w, p, l, k}(t), \forall w, p, k, m ; i \notin\{r, s\}
$$

Third, flow propagation constraints entail:

$$
\begin{gathered}
x_{m}^{w, p, l, k}(t)=E_{m}^{w, p, k}\left(t+\tau_{m}^{l}(t)\right)-E_{m}^{w, p, k}(t)+\sum_{l^{\prime} \in p}\left\{x_{m}^{w, p, l^{\prime}, k}\left(t+\tau_{m}^{l}(t)\right)-x_{m}^{w, p, l^{\prime}, k}(t)\right\} \\
\forall l \in B(i) ; i \neq r ; w, p, k, m \\
\frac{d E_{m}^{w, p, k}(t)}{d t}=e_{m}^{w, p, k}(t), \forall w, p, k, m
\end{gathered}
$$

Fourth, nonnegativity constraints are:

$$
\begin{gathered}
x_{m}^{w, p, l, k}(t) \geq 0, \forall w, p, l, k, m \\
u_{m}^{w, p, l, k}(t) \geq 0, \forall w, p, l, k, m \\
v_{m}^{w, p, l, k}(t) \geq 0, \forall w, p, l, k, m \\
e_{m}^{w, p, k}(t) \geq 0, \forall w, p, k, m \\
E_{m}^{w, p, k}(t) \geq 0, \forall w, p, k, m
\end{gathered}
$$

Last, boundary and bike constraints include:

$$
\begin{gathered}
E_{m}^{w, p, k}(0)=0, \forall w, p, k, m \\
x_{m}^{w, p, l, k}(0)=0, \forall w, p, l, k, m
\end{gathered}
$$




$$
\begin{gathered}
\sum_{i} \Phi_{\text {bike }}^{i}(0)=Q_{\text {bike }} \\
\Phi_{\text {bike }}^{i}(t)=e_{\text {bike }}^{w, r}(t)-f_{\text {bike }}^{w, s}(t) \\
\Phi_{\text {bike }}^{i}(t) \geq 0
\end{gathered}
$$

\subsection{Variational inequality model}

The dynamic traffic flow pattern satisfying network constraint set (24)-(42) is indeed a multi-class generalised travel-disutility-based dynamic user equilibrium superpath choice state as described in the variational inequality problem (43).

$$
\sum_{k} \sum_{w} \sum_{p} \sum_{t} P U^{w, p, k^{*}}(t) \cdot\left(f^{w, p, k}(t)-f^{w, p, k^{*}}(t)\right) \geq 0
$$

where the feasible region is $\Omega=\{(24)-(42)\}$.

Theorem 1 There is at least one solution to variational inequality (43).

Proof. A standard theorem in the theory of variational inequalities indicates that: if the feasible region $\Omega$ is compact and the mapping function of the problem are continuous, there exists at least one solution to VI formulation (43).

First, as the feasible region $\Omega=\{(24)-(42)\}$ of VI formulation (43) consists of a set of linear constraints, thus the region is compact. Second, due to the imbalanced distribution of bikes at each TAZ, the travel disutility value during calculation is unsmooth, but the travel disutility function is still continuous. If the interval length is infinitely close to zero (i.e., continuous-time), the link inflows will be continuous with path inflows vector. Then the existence of the dynamic user equilibrium solution can be guaranteed in principle.

Due to the inclusion of 0-1 integer variables $\delta^{w, p, l, t^{\prime}, k}(t)$, the disutility calculated by Eq. (3) are non-linear and non-convex. In addition, strict monotonicity of the path disutility is not ensured as it is highly dependent on the structure of supernetwork. Therefore, the solution uniqueness of VI formulation (43) cannot be guaranteed.

In real situation, the time interval length is hardly to be infinitely close to zero. The violation of the solution existence requirement might lead to the non-existence of solution. Moreover, the unbalanced distribution of demand and supply will lead to the unsmooth property of travel disutility function. As of the discrete time assumption, the disutility value may also not be small enough to ensure the existence of the proposed dynamic user equilibrium. Thus, we adopt the tolerance-based dynamic user equilibrium principle proposed by Szeto (2003), and therefore formulations (21)-(23) and (43) could be converted as:

$$
\left\{\begin{array}{l}
P U^{w, p, k}(t) \leq(1+\varepsilon) \cdot \Gamma^{w, p, k^{*}}(t), \text { if } f^{w, p, k}(t)>0 \\
P U^{w, p, k}(t) \geq(1+\varepsilon) \cdot \Gamma^{w, p, k^{*}}(t), \text { if } f^{w, p, k}(t)=0
\end{array}, \forall w, p, k, t\right.
$$




$$
\sum_{k} \sum_{w} \sum_{p} \sum_{t} \max \left\{P U^{w, p, k *}(t),(1+\varepsilon) \cdot \Gamma^{w, p, k *}(t)\right\} \cdot\left(f^{w, p, k}(t)-f^{w, p, k *}(t)\right) \geq 0(45)
$$

where the feasible region is $\Omega=\{(24)-(42)\}$. When $\varepsilon=0$, (45) is equivalent to (43). When $\varepsilon>0$, it is reasonable in real situation, where the travel disutility of all used paths between the OD pair are within an acceptable tolerance from the minimum OD path disutility.

Inference 1 There is at least one solution to variational inequality (45).

Proof. Szeto (2003) has proved that the tolerance-based principle is a generalisation of the traditional dynamic user equilibrium principle. That is, if $\varepsilon=0,(45)$ will be reduced to (43). It is a relaxation of (43), so the solution exists.

\subsection{Solution algorithm}

A path-flow swapping algorithm is developed to solve the proposed model. The solution algorithm starts with initialised flows on the feasible path and available bikes in each TAZ. The detailed steps are described as follows:

Step 0: Set up the parameters and load the multi-state super networks for all modes, $\mathrm{t}=1$.

Step 1: Identify the feasible path sets using enumeration method, in which the paths longer than 3 times of the shortest path or with superfluous transfer times are excluded.

Step 2: Load the demand at time $t$ on the network.

Step 3: Assign the demand stochastically on feasible paths, in which the constraints (28)-(29), (33)-(37), and (38)-(42) are satisfied.

Step 4: Calculate the real time traffic volume on each link at time $t$ and bike supplies at each node based on the constraints (24)-(27) and (30)-(32).

Step 5: Calculate the travel time, monetary cost and comfort loss on each link based on Eqs. (5)-(20).

Step 6: Calculate the travel disutility on each link based on Eq. (4).

Step 7: Calculate the travel disutility on each feasible path based on Eq. (3).

Step 8: Convergence test. If the following convergence condition $\max \left(\boldsymbol{\mu}^{\mathbf{a}} \cdot\left(\mathbf{P} \mathbf{U}^{\mathbf{a}}-P U^{* a}\right)\right) \leq \varepsilon \cdot P U^{* a}$ is satisfied, the algorithm stops, record the traffic volume on the network at time $\mathrm{t}$ ', update the number of bikes in each TAZ, update $t$, $t=t+1$, and return to Step 2; otherwise, continue. Herein, $\boldsymbol{\mu}^{\mathbf{a}}$ and $\mathbf{P} \mathbf{U}^{\mathbf{a}}$ denote the vectors of traffic flow indicator and path disutility at iteration $a$ respectively, $P U^{* a}$ is the minimum path disutility at iteration $a$.

Step 9: Update current traffic flows, using:

$$
\mathbf{f}^{\mathbf{a}+\mathbf{1}}= \begin{cases}\max \left(0, \mathbf{f}^{\mathbf{a}}-\rho^{a} \cdot \mathbf{f}^{\mathbf{a}} \cdot\left(\mathbf{P} \mathbf{U}^{\mathbf{a}}-P U^{* a}\right)\right), \text { if } w, p, t, t^{\prime} \notin \Lambda^{a} \\ \mathbf{f}^{\mathbf{a}}+\frac{\sum_{w, p, t t^{\prime} \notin \Lambda^{n}}\left(\mathbf{f}^{\mathbf{a}}-\mathbf{f}^{\mathbf{a}+\mathbf{1}}\right)}{\left|\Lambda^{a}\right|}, & \text { if } w, p, t, t^{\prime} \in \Lambda^{a}\end{cases}
$$


where $\Lambda^{a}=\left\{w, p, t: P U^{w, p, k, t^{\prime}}(t)^{a} \leq(1+\varepsilon) \cdot P U^{* a}\right\}$, and $\rho^{a}=\rho^{0} /\left(a / \gamma^{0}\right) \cdot \rho^{a}$ and $\gamma^{0}$ are given as flow adjustment parameters; $\mathrm{f}^{\mathrm{a}}$ is the path flow vector at iteration $a$. Considering the constraints (40)-(42), update the number of bike at each TAZ, $a=a+1$, return to Step 4.

During the path-flow adjustment process, the manipulated conditions, $\lim _{a \rightarrow \infty} \rho^{a}=0$, $\lim _{a \rightarrow \infty} \rho^{a} \cdot f^{w, p, k, t^{\prime}}(t)^{a}=0$ and $\sum_{a} \rho^{a} \cdot f^{w, p, k, t^{\prime}}(t)^{a}=+\infty$ (Nagurney and Zhang, 1997; Huang and Lam, 2002) hold and direct the process to an convergence state within tolerance limit.

\subsection{Profit analysis}

One intrinsic question arises is whether the operator can still gain profits or not after introducing the proposed pricing strategy. We consider a general profit definition, which includes not only the fare revenue, but also the user value. User value is important when a new service enters the market. Operators might be willing to lose money at the initial stage of service operation if they can attract more users and dominate the market. After the initial promotion stage, the operator can adjust the operational goal to pursue fare revenue maximisation. Therefore, a set of comprehensive profit optimisation model is developed for both initial promotion stage and the normal operation stage. At the initial promotion stage, the main decision variable is the total bikes investment, i.e., what is optimal bike supply to the market, while the main operational goal is to attract maximum number of users with limited resources. Therefore, the maximisation profit objective function could be expressed as:

$$
\max \Upsilon\left(\mathrm{Q}_{\text {bike }}\right)=\varpi Q_{\text {user }}+\pi Q_{\text {user }}-\psi Q_{\text {bike }}
$$

where $\Upsilon$ is the overall profit, and $Q_{b i k e}$ is the optimal number of bikes, $Q_{\text {bike }}=\sum_{i} \Phi_{b i k e}^{i}(0) \cdot Q_{u s e r}$ is the number of registered users who have paid the deposit. The first item on the right-hand side describes the total user value in the market, where $\varpi$ is the unit user value. The second item represents the total deposit revenue, where $\pi$ is the deposit per user. The third item depicts the total bike cost, where $\psi$ is the cost per bike. The constraints of model objective function (46) are (3)-(42).

After the initial promotion stage, there will be a huge number of stable existing users in the market. Few potential new users will be attracted, even though the price promotion continues. Operators could adjust the operational goal to achieve fare revenue maximisation. At this normal operation stage, the bike investment and new deposit income are nearly zero due to the few new users. In this case, the operators seek to find out the optimal pricing scheme and bike supplies so as to maximise the total profit, wherein the objective function could be expressed as:

$$
\max \Upsilon\left(\Phi_{\text {bike }}^{\mathrm{i}}(0), \mathrm{G}_{\text {bike }}(t)\right)=\bar{\pi} Q_{\text {user }}+G_{\text {fare }}-\bar{\psi} Q_{\text {bike }}
$$


The first item on the right side is the profit from user deposit, where $\bar{\pi}$ is the interest from one user deposit. $Q_{u s e r}$ is the number of registered users who have paid the deposit. The second item is the fare revenue, where $G_{\text {fare }}=\sum_{t} \sum_{w} \sum_{p} \sum_{k} f_{b i k e}^{w, p, k}(t) \cdot G_{\text {bike }}(t)$ and $f_{b i k e}^{w, p, k}(t)$ is the dynamic traffic equilibrium solution of (42) that is related to $G_{b i k e}(t)$ and $\Phi_{b i k e}^{i}(t)$. The third item covers the bike depreciation expense and operation cost, which is assumed to be positively related to the number of bikes, $Q_{\text {bike }}=\sum_{i} \Phi_{\text {bike }}^{i}(0)$. The constraints of objective function (47) are (3)-(42).

The primary objective of the above analysis is to present model formulation for the dockless bike sharing service providers to find the optimal operation strategy if the proposed negative pricing scheme is applied. Two formulated models are introduced for different stages of service operation as the service providers' objective changes as time advances. One can notice that, the forumated models are indeed in the format of bi-level programming formulation, which is very hard to be solved to its global optimal solutions. In this study, while we propose this model formulation on finding optimal operation strategy in presence of negative pricing scheme, the development of the efficient solution algorithms to obtain quality solutions is left to be addressed in the future studies. In practice, when high solution quality is not required, one can be recommended to apply sensitivity analysis approach to obtain a good solution result.

\section{Numerical examples}

Two numerical examples are presented to illustrate the effect of the proposed negative pricing strategy on bike relocation, travel behaviour and network performance.

\subsection{Numerical results for test example 1}

The first example is designed to show the effect of the proposed pricing strategy onto achieving a more balanced bike relocation. For illustration purpose, we conduct the numerical example in a simple network with three nodes and links as shown in Figure 5. The number on each link represents the travel distance. Traffic flow over time is uniformly distributed in the network as shown in Table 3, where the node in business area only has inflow demand, the node in residential area only has outflow demand, and the rest node at metro station has both inflow and outflow demand. Average speed, bus ticket price, headway information and a series of discomfort conversion parameters for different modes are set to be the same as in Meng et al. (2014). VOT parameters are adopted from Zhang et al. (2017). Bi-directional flow travel time equation is adopted from $\mathrm{Wu}$ and $\mathrm{Lam}$ (2003). Other parameters are set as follows: $k=1$, VOT $=10, \varepsilon=0.05$, $\Phi_{\text {bike }}^{i}(0)=50, \quad \Phi_{t h, 1}^{i}(t)=5, \quad \Phi_{\text {th, }}^{i}(t)=3, \quad g_{\text {bike }}^{\text {neg }}(t)=0.5, \quad g_{\text {bike }}^{\text {pos }}=0.5, \mathrm{~T}=70 \mathrm{~min}$ and $\Delta=1 \mathrm{~min}$. Traffic departures from 0 to $60 \mathrm{~min}$. 

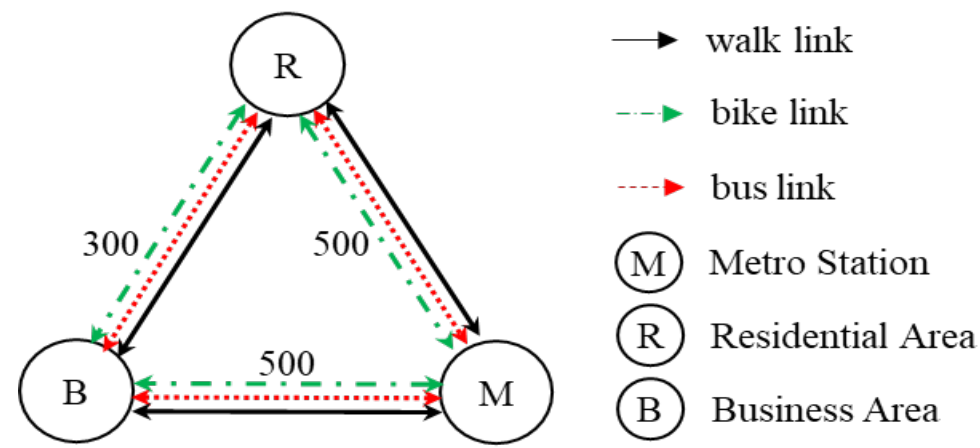

Figure 5 Example 1 network

Table 3 OD demand of example 1

\begin{tabular}{|l|r|r|r|}
\hline O D & Business Area & Residential Area & Metro Station \\
\hline Business Area & 0 & 0 & 0 \\
\hline Residential Area & 1 traveller $/ \mathrm{min}$ & 0 & 2 travellers $/ \mathrm{min}$ \\
\hline Metro Station & 1 traveller $/ \mathrm{min}$ & 0 & 0 \\
\hline
\end{tabular}

Figure 6 shows the number of bike trips and bike trip accumulation at metro station over time. Three different pricing strategies, i.e., negative pricing, free pricing and normal positive pricing, are tested and compared in this example. One can observe that the number of bike trips under three pricing strategies are identical, as well as the bike trip accumulation. It is because the travel disutility of bike is the lowest as compared to other alternative modes. In this study, while the travel disutility is determined by many factors including travel time, monetary cost, and comfort loss, comfort loss, the differential monetary cost under the three pricing strategies would not change the fact that cycling is the most favourite travel mode in this tested example. However, the dynamic and spatial bike departures and arrivals, as well as the bike supply in the network, are significantly different under different pricing strategies, as will be analysed in the following discussions.

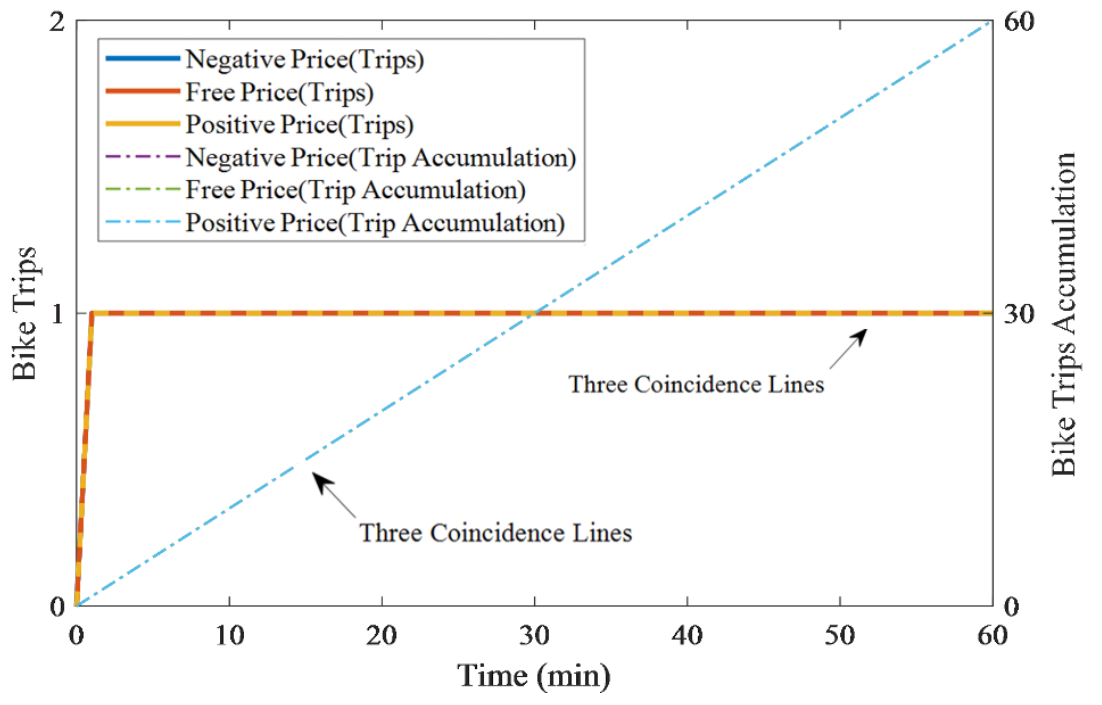


Figure 6 The number of bike trips and bike trip accumulation departure from metro station over time

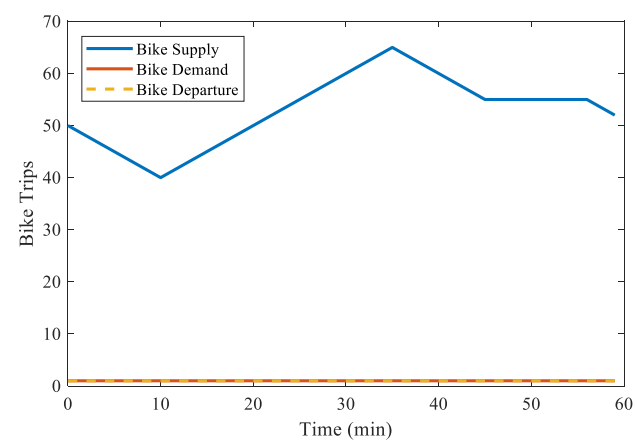

(a) Negative Price

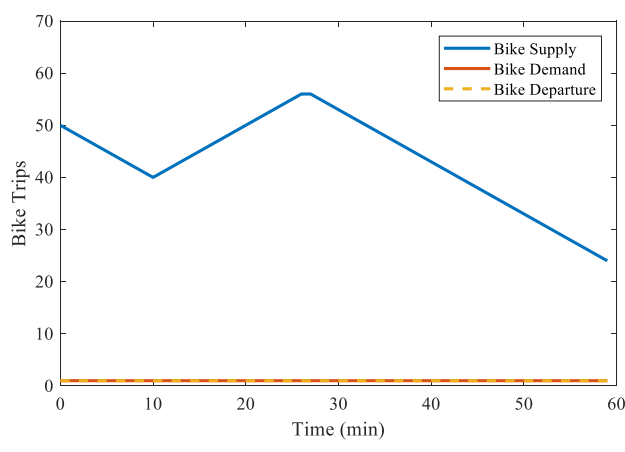

(b) Free Price

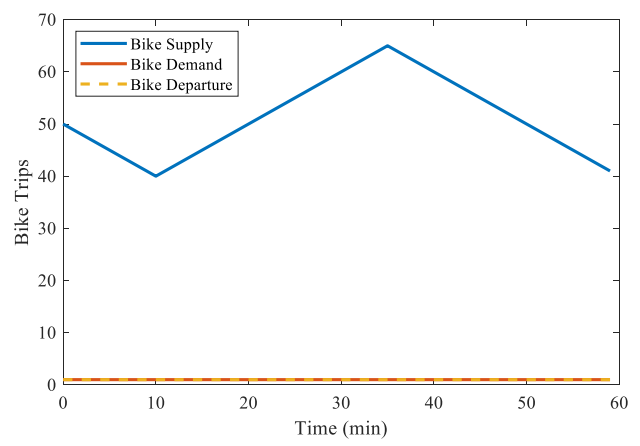

(c) Positive Price

Figure 7 The detailed supply, demand and actual trip numbers at metro station over time

The detailed supply, demand and actual bike trip numbers are given in Figure 7. One can observe that the supply is always above the real demand. However, the supply lines under three different pricing strategies are different. The number of available bikes continuously decreases at the beginning stage. As the time advances, the number of inflow bike arrivals would exceed the outflow bike departures and the number of bikes starts to increase. The arrival rates at the metro station under different pricing strategies are displayed in Figure 8. As the initial number of bikes in residential area is limited, the bikes that flow from residential area to metro station would reduce to zero and the number of bikes at metro station would decrease. It should be noted that, the time points when the bike supply starts to switch from increasing to decreasing for the three pricing strategies are different. The switching time under NP or PP is later than that with FP. According to Figure 8, the number of bike arrivals under FP is less than that for NP and PP. Specifically, the number of bike arrivals under NP does not continuously decrease as under PP, due to the extra bike arrivals stimulated by NP. 


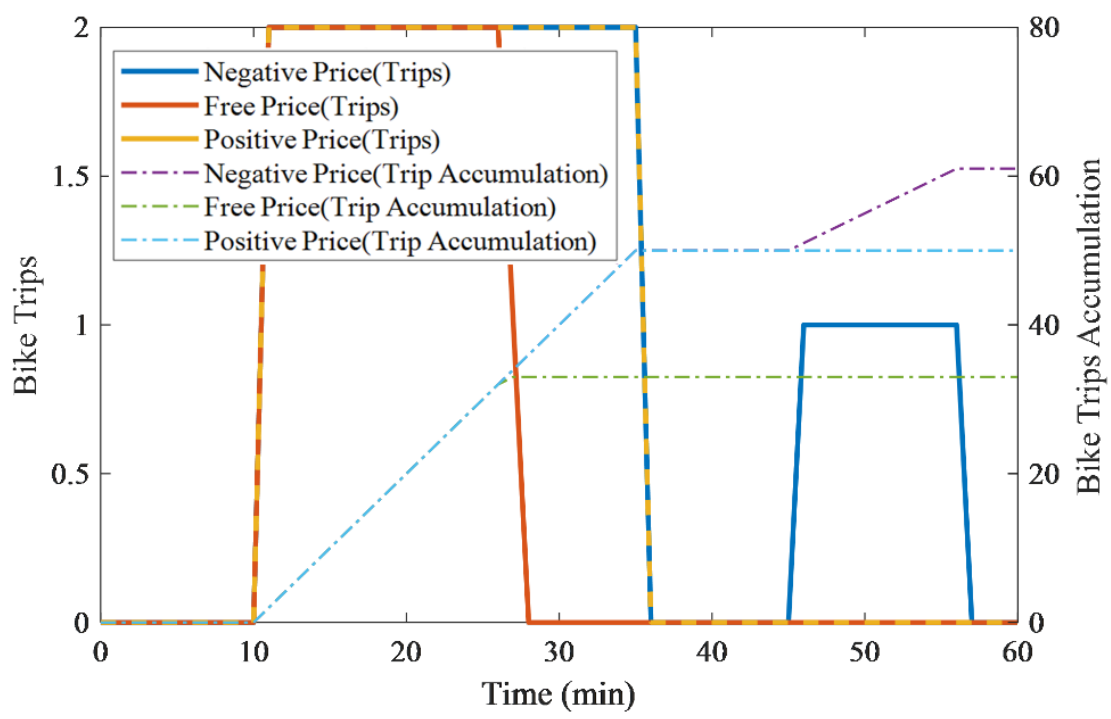

Figure 8 The number of bike trips and bike trip accumulation arrival at metro station over time

Next, we want to further analyse the extra bike arrivals when negative pricing scheme is applied. As in Table 3, the demand from node B to node $\mathrm{M}$ is 0 . The new bike arrivals must be from node R. Figure 9 shows the departure and arrival information at node R. One can notice that the bike departure from node $\mathrm{R}$ is still positive, while at the same time there are no available bikes under FP and PP at node R. The total bike departures are even higher than the initial supply of 50 bikes. As $\mathrm{R}$ has been set as an outflow node, the extra bikes at node $\mathrm{R}$ demonstrate that some travellers have changed their travel behaviour to park bike at node R under NP and the bike is later used by another traveller from node $\mathrm{R}$ to node $\mathrm{M}$. All of these have clearly illustrated the effectiveness of the negative pricing in terms of a more balanced bike relocation.

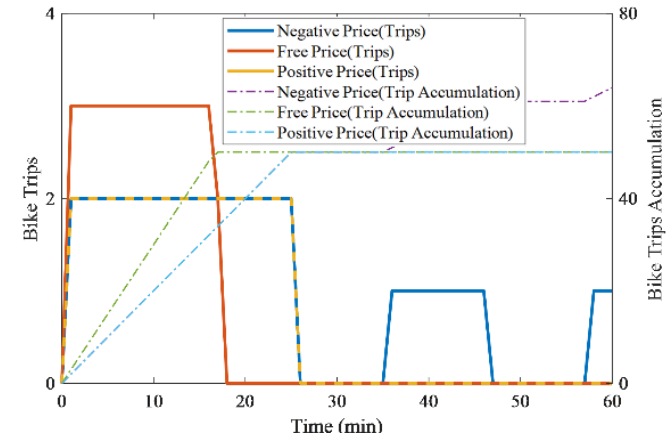

(a) Departure

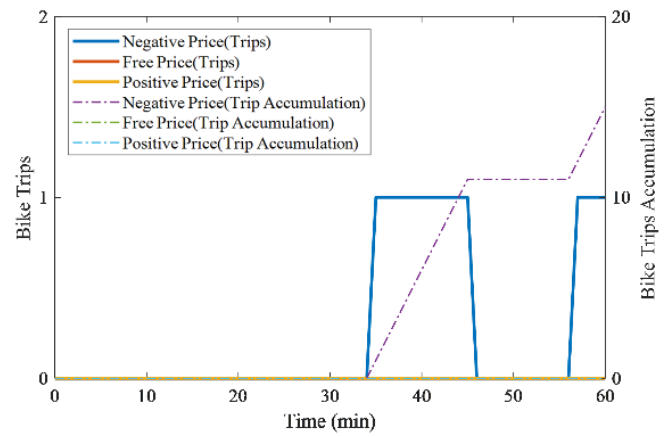

(b) Arrival

Figure 9 The number of bike trips and bike trip accumulation at residential area

It seems that FP scheme could be a good substitute of NP scheme as free charging is able to attract bike users and requires no additional cost from operators. The results from this numerical example can provide some direct comparisons between the two pricing strategies. Figure 9 can explain why the number of bike arrivals under FP at node $\mathrm{M}$ is smaller than that under NP. Under FP, the number of travellers using bike sharing is more than it under NP. Travellers who originally walk from node $\mathrm{R}$ to node B will change to use bike under FP. Under NP, as the node B is in the status of 
oversupply, there is no monetary reward from node $\mathrm{R}$ to $\mathrm{B}$. Those travellers will remain to walk from node $\mathrm{R}$ to node $\mathrm{B}$. However, according to Table 3, there are positive travel demand from node $M$ to node $B$. The supply at node $M$ is sufficient at the very beginning. Thus, there is positive bike flow from node $\mathrm{M}$ to node $\mathrm{B}$ under FP. However, under NP there is no bike arrival at node B at certain time intervals (e.g., from 35 to 45 and from 57 to 60 ). This is because traveller has changed his/her path to park the bike at node R, according to Figure 9 and Figure 10. In other words, under NP, traveller will cycle from $\mathrm{M}$ to $\mathrm{R}$ and change to other modes to arrive at $\mathrm{B}$, due to triggered negative price from node $\mathrm{M}$ to node $\mathrm{R}$. For travellers, although the total trip distance and total travel time increase, the monetary reward from NP can offset the increased disutility. For operators, travellers contribute to achieve a more balanced bike relocation, which can save the operation cost substantially.

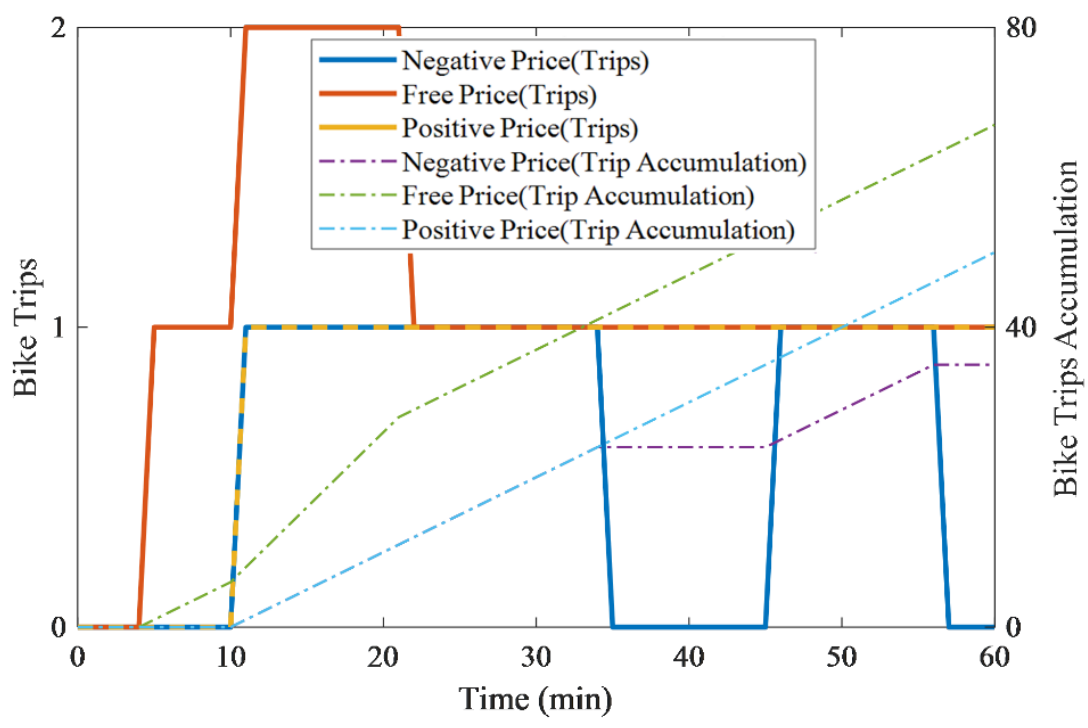

Figure 10 The number of bike trips and bike trip accumulation arrival at business area

Figure 11 shows the bike flow evolution on each link over time. First, under PP, there are bike flows only on link M-B and link R-M, which is equal to the demand according to Table 3. The demand from node $\mathrm{R}$ to node $\mathrm{B}$ does not select to cycle because the short trip distance is more suitable for walking. As of the sufficient supply at node $\mathrm{M}$, the bike flow on link M-B is stable. Since the supply at node $\mathrm{R}$ is not sufficient, when all bikes are used, the link flow R-B becomes to zero. Second, under FP, bike flow on link M-B does not change over time. It is also because of the sufficient supply at node M. The bike flow on link R-M is similar to the situation under PP, which first increases to peak value, then stabilizes for a while before it decreases in the end. The stable period at peak value in Figure 11(d) under FP is shorter than that under PP due to the bike flow on link R-B as shown in Figure 11(c). This is in line with the above conclusion obtained from Figures 9 and 10. It reflects that when the number of shared bikes is limited, FP could only speed up the bike usage rate but further enlarge the gap between supply and demand at the same time, which indeed cannot improve the service quality of the bike sharing system. Furthermore, FP attracts the short-distance travel demand, which is more suitable for walking. It is indeed a waste of resources and can even deteriorate the level of service. Last, when NP implemented, bike flow on link M-B fluctuates over time as shown in Figure 11(a), which indicates the successful bike relocation and 
repeated use of bikes within the peak hour. Basically, NP attracts and diverts some bike flow on link M-B to link M-R to support the bike supply at node R. Compared to FP, NP induces higher utilization rate for the shared bikes through a more balanced relocation achieved by the bike users. Also, NP does not increase the traffic flow in the network.

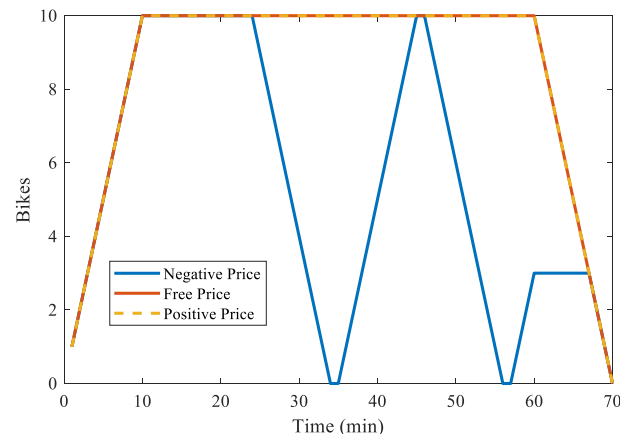

(a) Link M-B

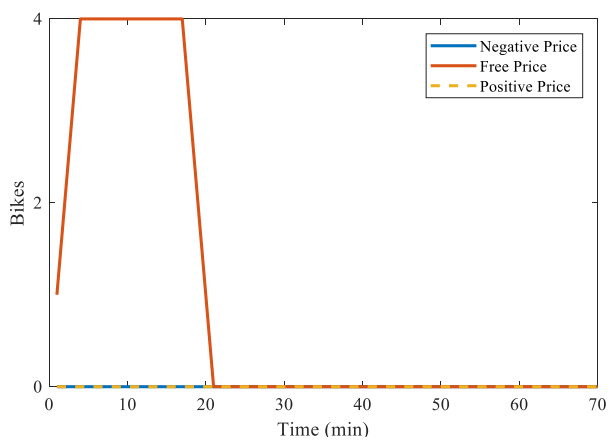

(c) Link R-B

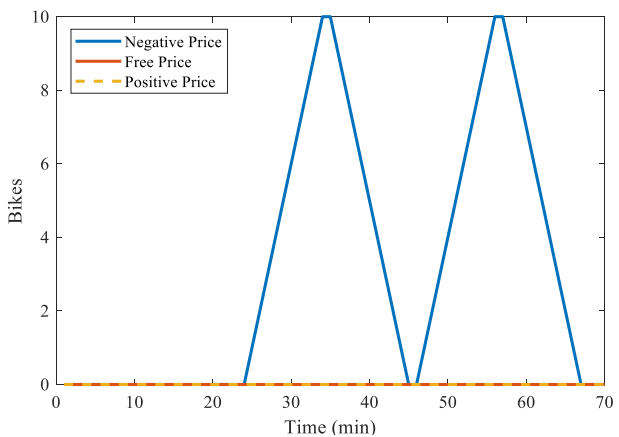

(b) Link M-R

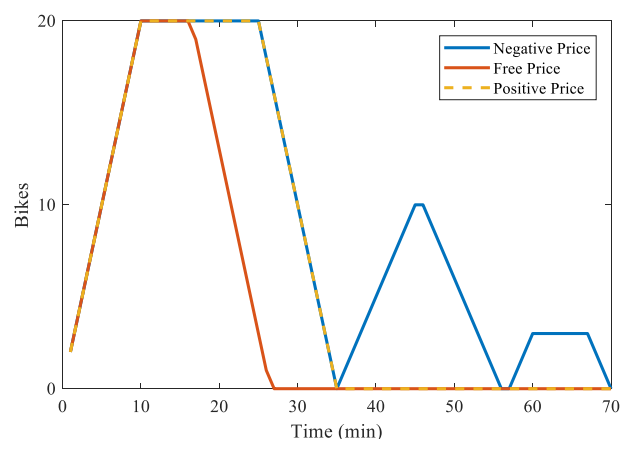

(d) Link R-M

Figure 11 The bike flow changes on each link over time

In addition, we conduct the sensitivity analysis of the threshold values and the results are illustrated in Figure 12(a) and (b). Firstly, we assume a fixed threshold value at origin node and seek to know how the threshold value at destination node affects the system performance in terms of total trip number and fare revenue. From Figure 12, one can observe that, as threshold value at destination node increases, the NP condition tends to be fulfilled more easily. Thus, the total number of trips goes up while the total fare revenue grows up first and then reduces due to the large amount of negative reward. If maximum fare revenue is to be achieved, the optimal threshold value at destination node could be easily obtained from the sensitivity analysis. In addition, we want to find how threshold value at trip origin node would affect the service operation performance. To do so, we assume fixed threshold value at destination node. It can be observed that, when threshold value at origin node increases, the NP condition becomes more difficult to be satisfied, and thus the total bike trips and the total fare revenue reduce. 


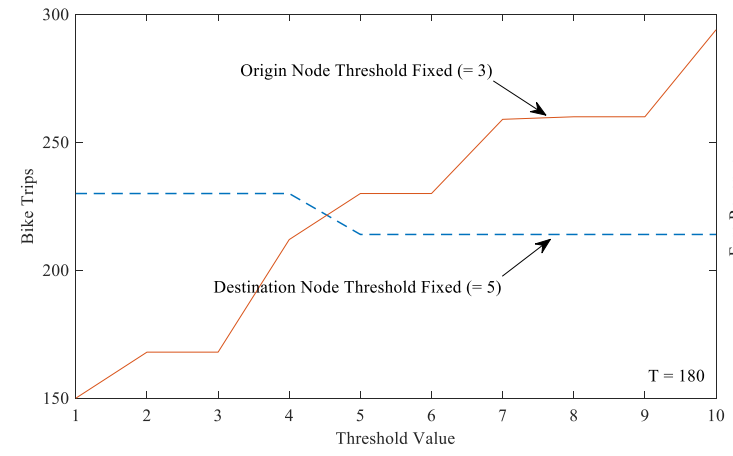

(a)

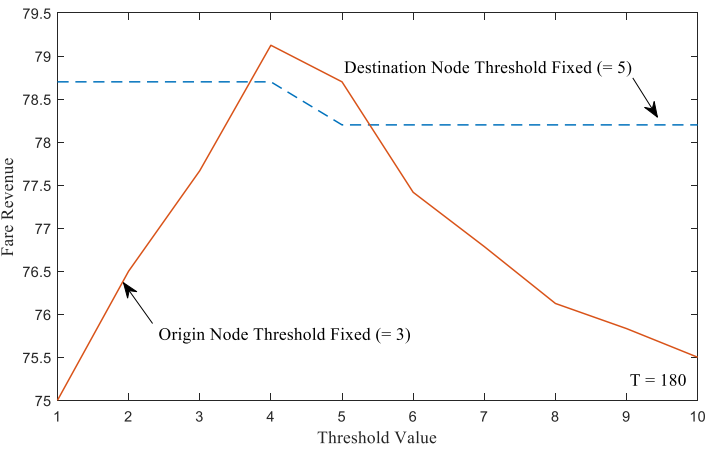

(b)

Figure 12 The influence of threshold values on bike trips and fare revenue

The previous analysis is based on the parameter setting that VOT equals to 10 dollars per hour for all travellers. Figure 13 is depicted to show how the total amount of bike trips and fare revenue change after different VOT values. As VOT reduces, more travellers prefer to using bike. The increasing bike demands would result in more negative reward and thus renders the total fare revenue first increases before it goes down. In example 1, when all the travellers have the same VOT (VOT=10), the total bike trips are 230 units and the total fare revenue is 78.7 units. When we consider different classes of travellers, as categorized by different VOT values (VOT=20, 10, 7), we can obtain the model results that the total amount of bike trips is 236 and the total fare revenue is 79.6 .

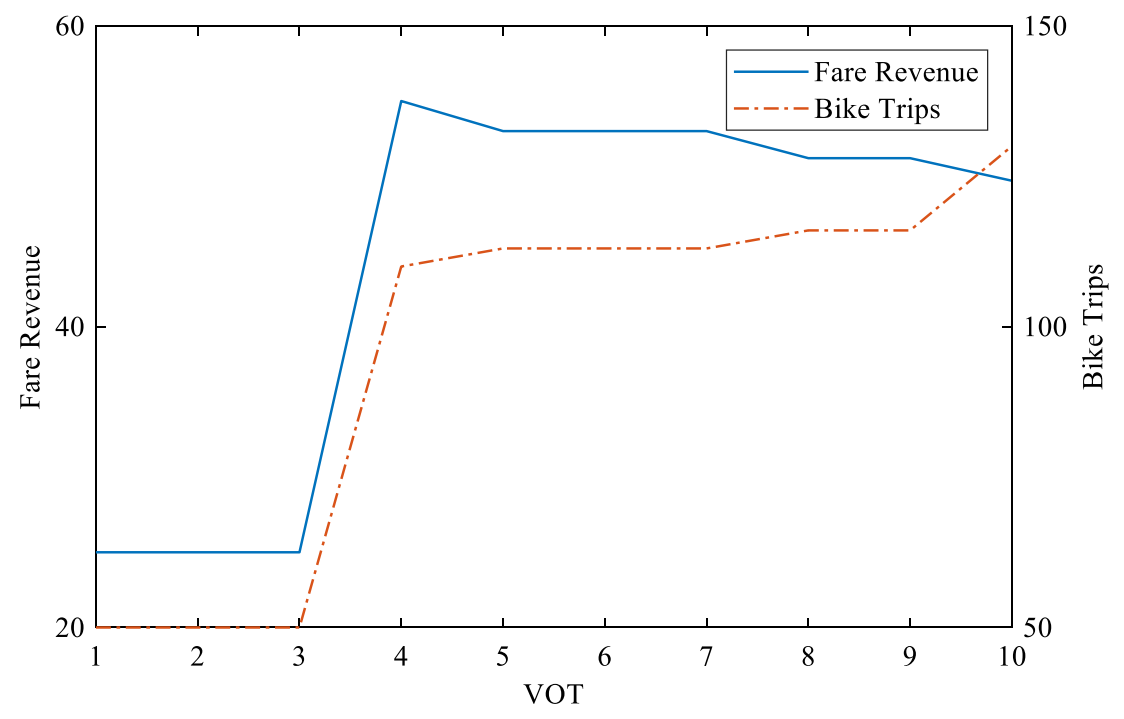

Figure 13 The influence of VOT on bike trips and fare revenue

\subsection{Numerical results for test example 2}

The second example is designed to illustrate the performance of NP in a more complicated network. A benchmark network Sioux Falls network is applied. The network parameter and population data are extracted from Chakirov and Fourie (2014). Three type of land use characteristics are classified: residential area, business area, and 
metro station as shown in Figure 14. The traffic flow among OD is calculated based on Gravity model in Table 4. Assume the departure time are randomly distributed within the range of $[1,60]$ minutes, $\mathrm{T}=75, \varepsilon=0.05, k=3$. Three types of VOTs are considered with the value of 20,10 and 7. Other parameters are same as above.

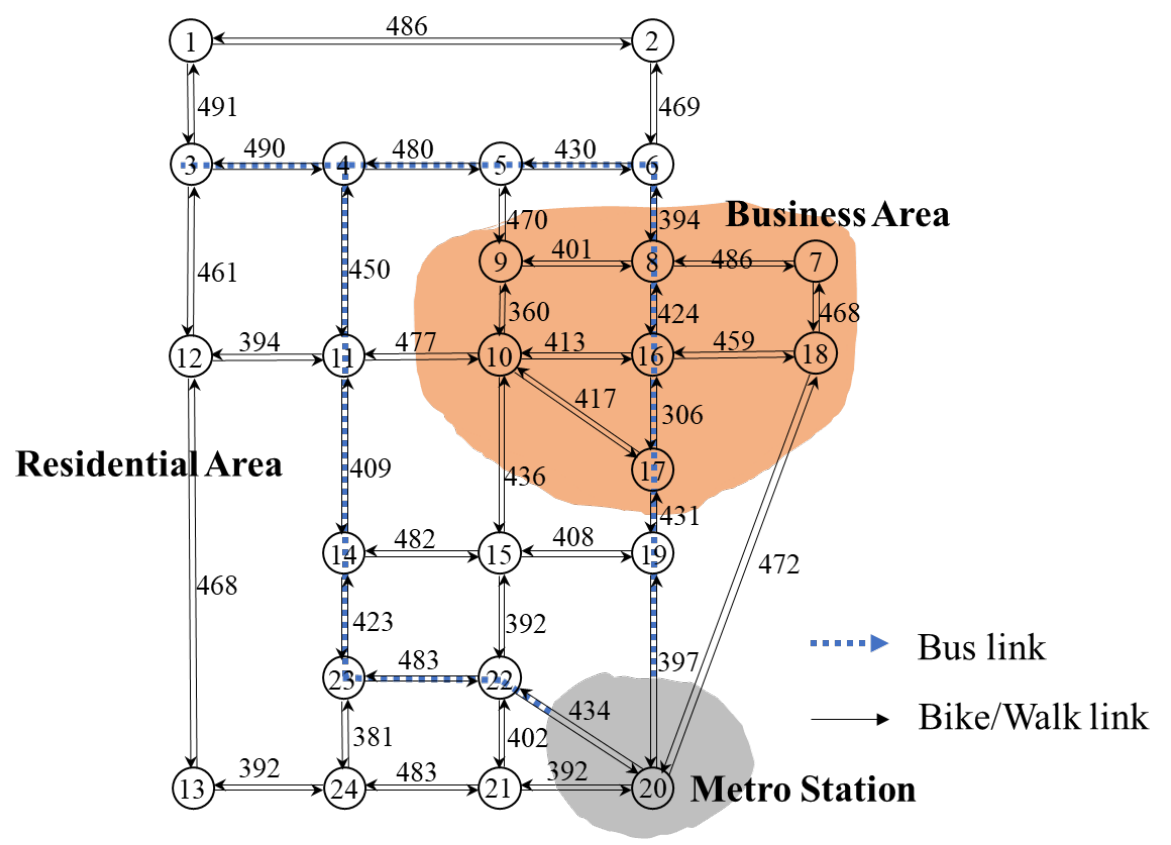

Figure 14 Simplified Sioux Falls network (the numbers on link denotes the link distance)

Table 4 OD demand of example 2

\begin{tabular}{|l|r|r|r|}
\hline O D & Business Area & Residential Area & Metro Station \\
\hline Business Area & 0 & 0 & 0 \\
\hline Residential Area & 2140 travellers & 0 & 4291 travellers \\
\hline Metro Station & 3541 travellers & 0 & 0 \\
\hline
\end{tabular}

The computational experiments are run in MatlabR2017b on a notebook with an Intel Core i5-4210U, 1.7 GHz under Windows 10 Professional with 4 GB memory. When the program is complete, the running time for example 2 is 1943.07 seconds. Figure 15 shows the convergence of the solution algorithm. 


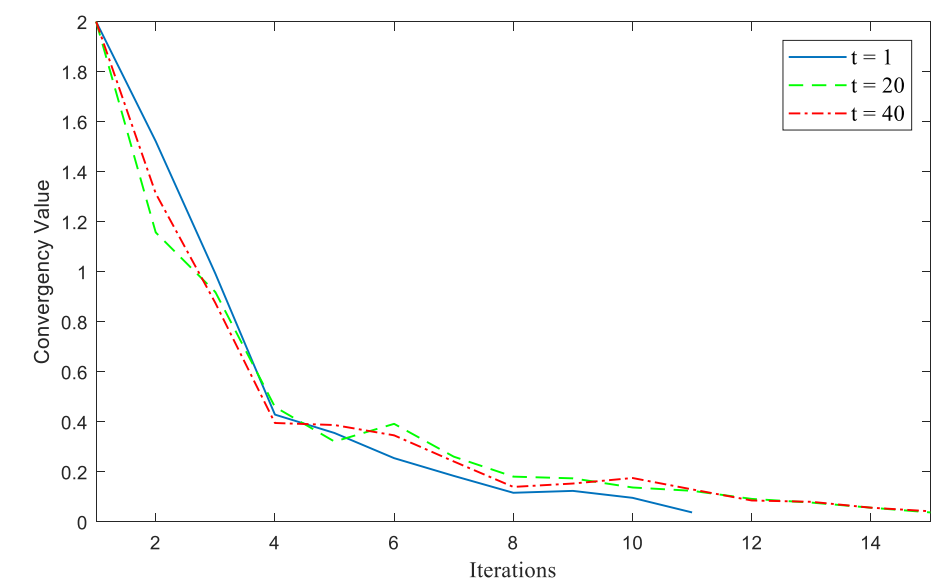

Figure 15 The convergence of the solution algorithm

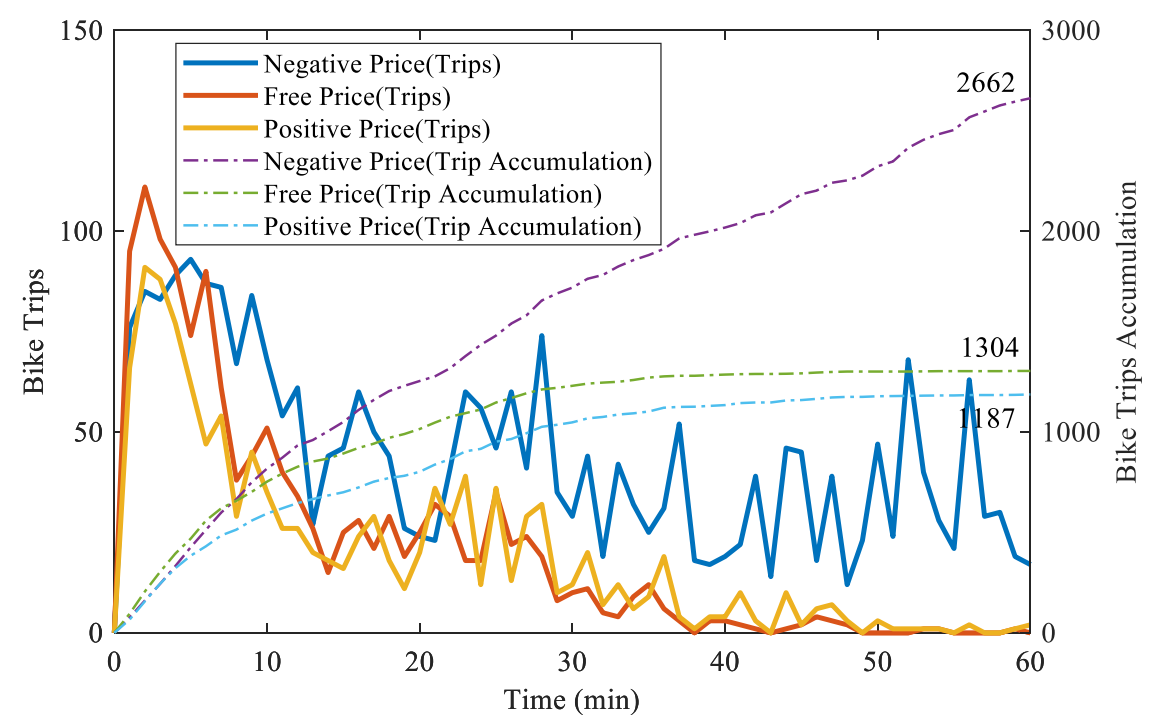

Figure 16 The number of bike trips and bike trip accumulation departure over time

Figure 16 shows the number of bike trips and bike trip accumulation in the network over time. At the beginning, the number of bike trips are quite high under all three strategies. When time advances, the bikes run out in high demand area, and the number of bike trips quickly drop under PP and FP. Consequently, the bike trip accumulation become stable under PP and FP. However, due to relocation feature of NP, bikes can be quickly re-supplied in undersupply area. The number of bike trips is always at a high level when NP is applied. The bike trip accumulation is even two times more than that under FP and PP.

Figure 17 presents the number of bike trips and bike trip accumulation in each type of area. As shown in Figure 17(a), the number of bike trips and bike trip accumulation in residential area have the same trend with Figure 16. Specifically, under FP, the number of bike trips is extremely high in the beginning and reduces to 0 after certain time period. Under NP, there are more bikes relocated from residential area, as can be observed in Figure 17(b). NP obviously attracts more travellers to park bikes at undersupplied residential area. More specifically, there are 11 bikes arriving at residential area under PP. The number is 56 under FP, and 593 under NP. According to Figure 16, the total bike usage is about 1000 under PP or FP. The 593 bikes will be reused by travellers 
from residential area to other places. The number of bike trips under NP has increased by about 50\%. Figures 17 (c) and (d) show the specific information at node 15 in residential area. 50 bikes run out quickly under FP or PP. However, about 230 bikes depart from node 15 under NP in the whole period, where 180 of them come from the relocation effect according to Figure 17(d).

Figures 17(e) and (f) give the number of bike trips and bike trip accumulation in business area. Figures $17(\mathrm{~g})$ and $(\mathrm{h})$ present the detailed information at one specific node in business area. According to Table 4, the demand in business area is 0 . The bike departure number under PP and FP are both zero. Under NP, there are about 600 bikes out of business area while the total bikes in the network are1200. It reflects that NP can achieve more than $50 \%$ bike relocation.

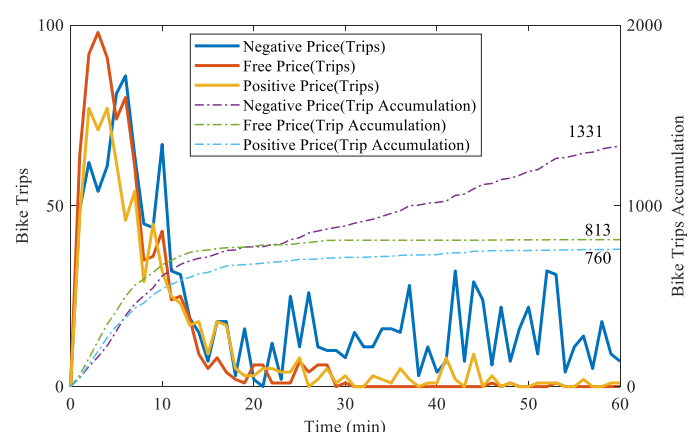

(a) Residential area departure

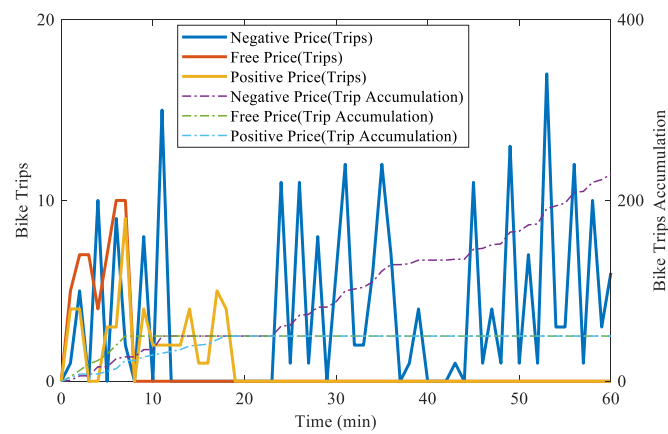

(c) Residential node 15 departure

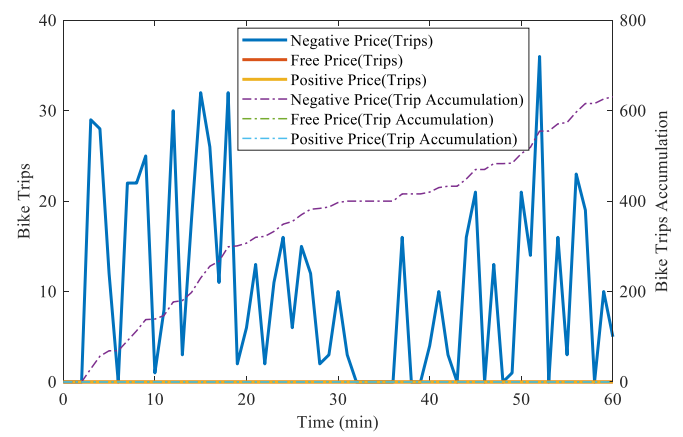

(e) Business area departure

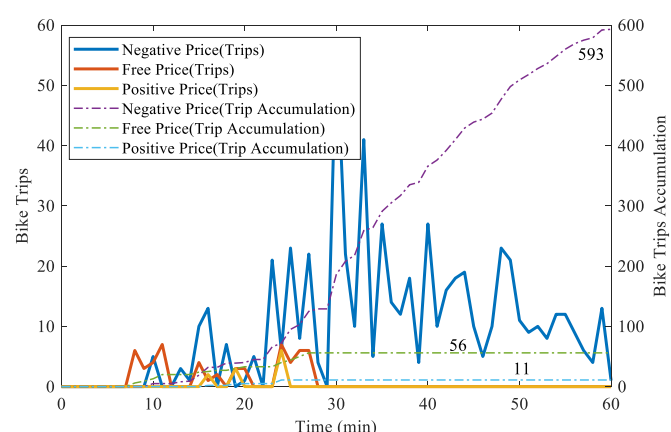

(b) Residential area arrival

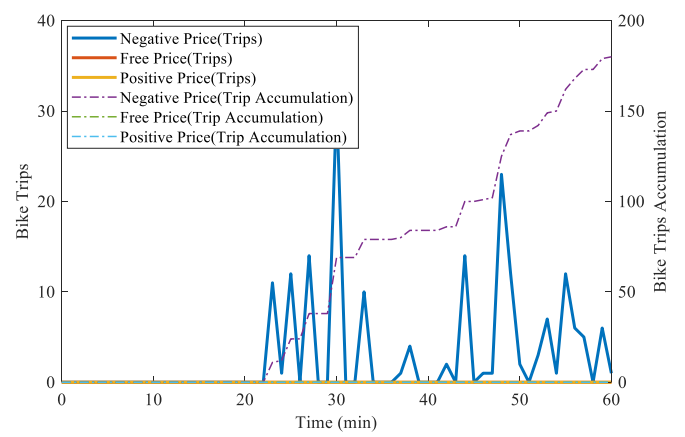

(d) Residential node 15 arrival

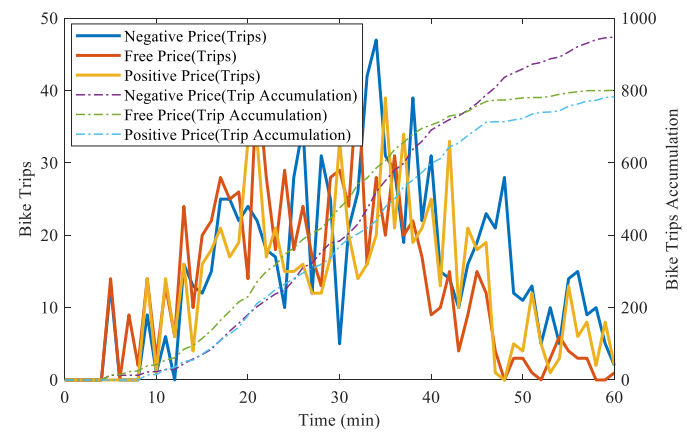

(f) Business area arrival 


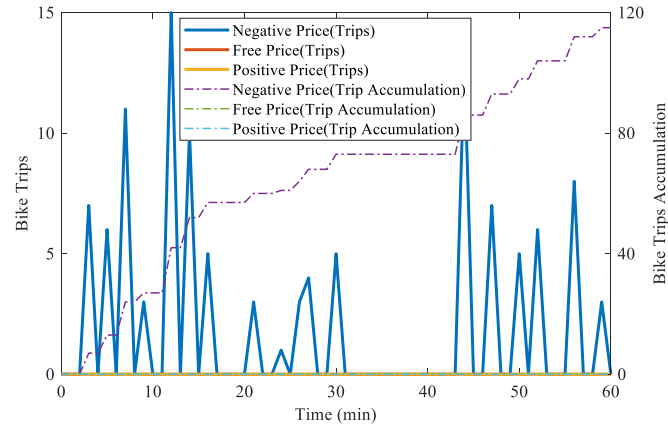

(g) Business node 8 departure

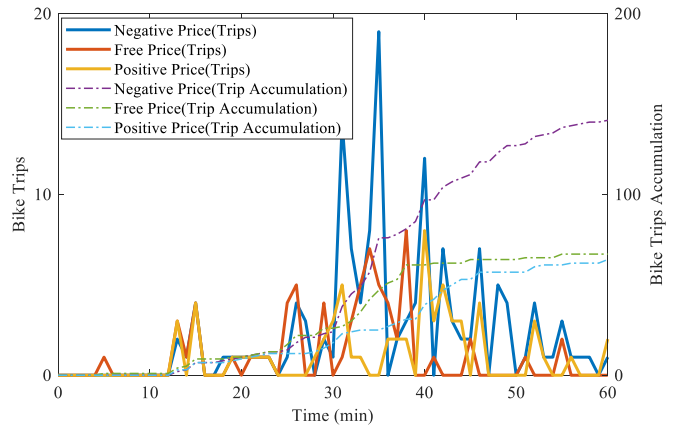

(h) Business node 8 arrival

Figure 17 The number of bike trips and bike trip accumulation over time

To describe the bike flow status in entire network, the dynamic bike flow rates on each link are calculated. The number of bike flow are divided into six groups: 1-40, 41-80, 81-120, 121-160, 161-200, and 201+. After classification, the results are shown in Figure 18. One can observe that FP easily leads to traffic congestion on bike links as it has the highest flow group (201+), as FP can quickly attract huge number of travellers to use bike. Although the total number of bike trips is similar with it under PP, the departure time of bike trips under FP is relatively concentrated. Under NP, though the total number of bike trips is greater than it under PP or FP, the trips are more evenly distributed in both time and space dimensions. Figure 19 shows the network performance under three pricing strategies. The average travel speed under FP is the slowest while the average travel speed under PP and NP are similar. The average travel distance under PP is the longest as the travellers who have paid the bike sharing service will fully utilise the bike to achieve door to door transport. Consequently, the number of average used links is maximum. Travellers might choose to cycle even if the trip distance is quite small under FP. Therefore, the average trip distance under FP is shorter than that under PP. Travellers will change their paths and modes under NP to relocate the bikes, which decreases the average trip distance. Indeed, the number of used links and the average trip distance are both the smallest for NP implementation.

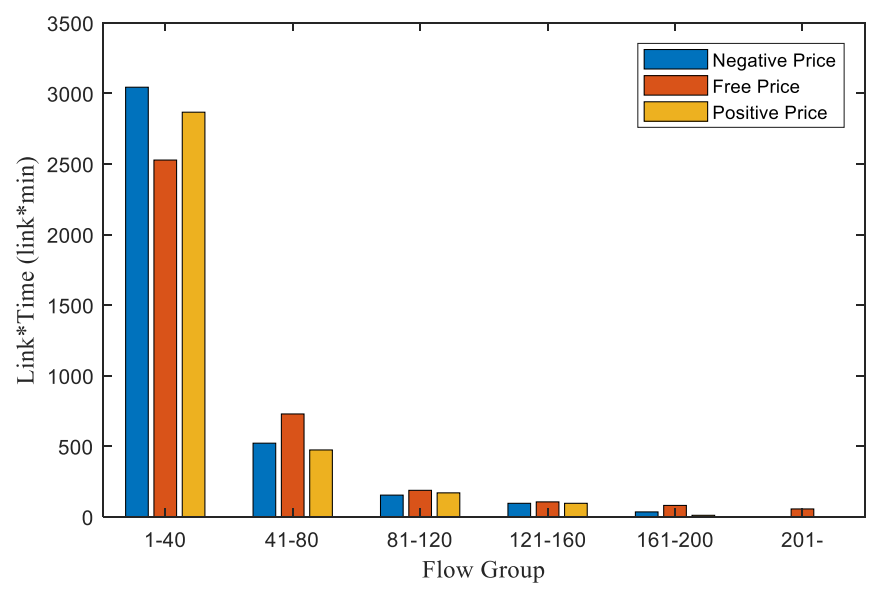

Figure 18 Link-time base on flow groups 


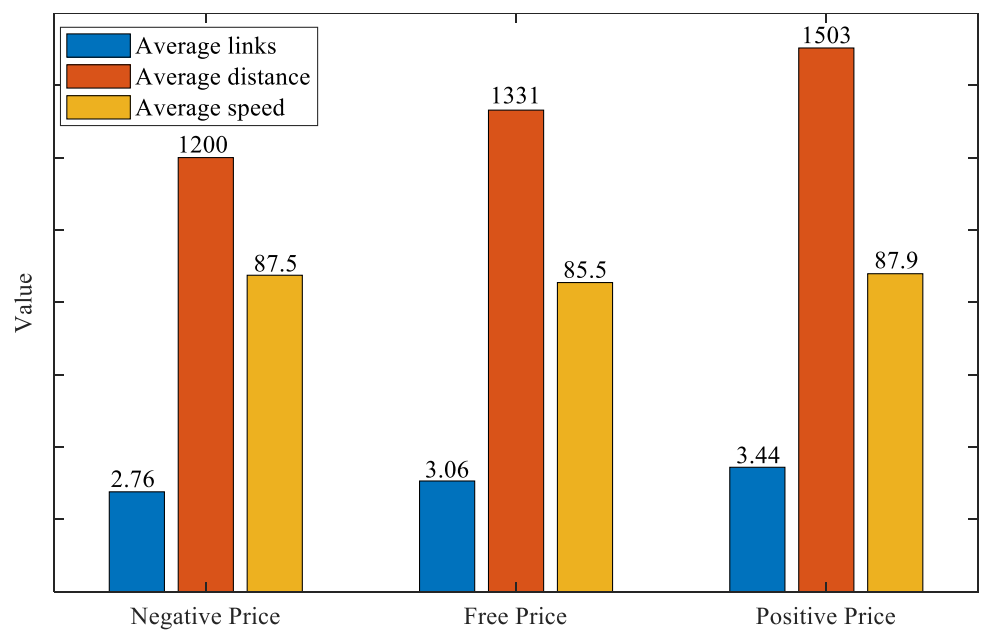

Figure 19 Network performance under three pricing strategies

Next, we would like to investigate the performances of the three pricing strategies from operators' perspective. Assuming there are 50 bikes at each node provided at the beginning, we plot the bike usage and fare revenue under different pricing strategies in Figure 20. Under FP, the total number of bike trips is only half of that under NP. Besides, the fare revenue is the least for FP, while the number of used bikes is maximum, which requires highest initial investment on bikes supply from operators. In this example, NP has better performance than FP in any performance indicator. Compared to PP, NP also performs better in terms of total bike trips. However, the fare revenue under NP is less than that under PP. It should be noted that the total fare revenue is parameter sensitive. In the proposed dynamic pricing strategy, the threshold value of negative price is determined by the real time ratio of supply to demand, which is a time related function Eq. (22). In this example, the threshold value is set as a fixed value $\Phi_{t h, 2}^{r_{\text {the }}}(t)=3$. If the parameter $\Phi_{t h, 2}^{r_{i k k}}(t)$ increases, higher fare revenue can be achieved, up to the requirement of the operators.

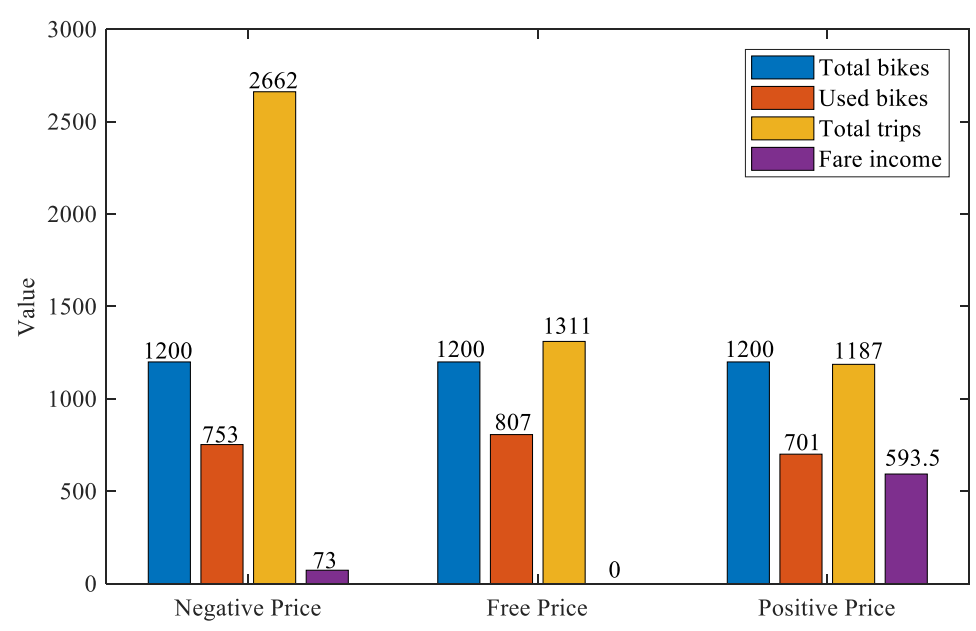

Figure 20 Bike usage and fare revenue under different pricing strategies

Next, we seek to find out the optimal number of bikes and fare revenue in initial promotion stage when different pricing strategies are applied. Some other parameters are set as follows: user value is $1000 \mathrm{RMB}$, deposit fee is $200 \mathrm{RMB}$ /user, bike cost is $1500 \mathrm{RMB} / \mathrm{bike}$ (CEIBS, 2017). The relationship between the number of bikes and 
fare revenue is shown in Figure 21(a). In this example, the fare revenue from FP is always 0 , which is the minimum among the three pricing schemes. The fare revenue from NP is less than PP in this example. The maximum objective function value under NP is indeed very close to that under FP. However, the required number of bikes is only 1127 with the budget of 1.17 million, which is only one third of the budget under FP.

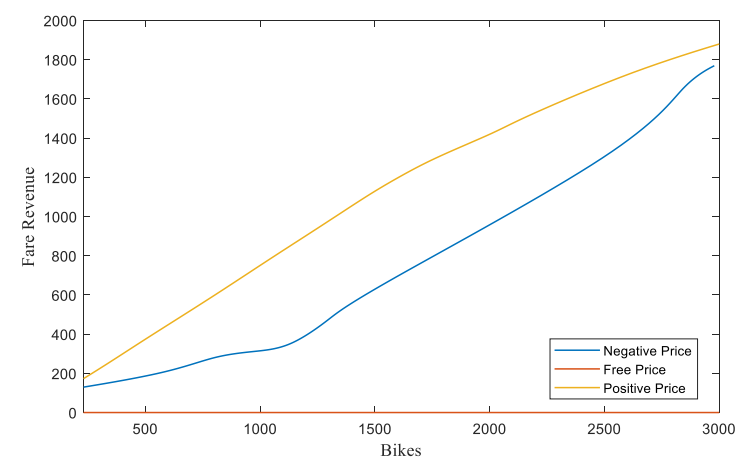

(a)

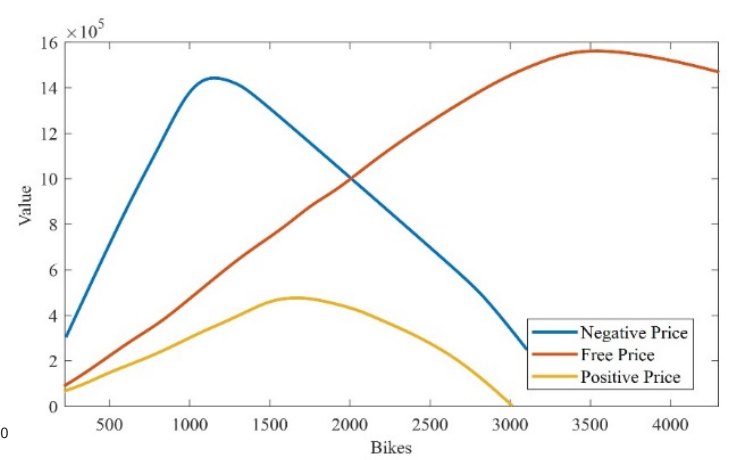

(b)

Figure 21 The relationship between the number of bikes and revenue and value

\section{Conclusions}

In this study, a new pricing strategy is proposed with negative price for dockless bike sharing service. Operator pay the users who cycle from the oversupplied area to undersupplied area. This pricing strategy could achieve bike relocation through stimulating travellers' behaviour changes. Travellers' choice behaviour is formulated by a user equilibrium dynamic traffic assignment model, in which traveller are allowed to use single or combined travel mode to complete the first/last mile trip by using walk, bike or bus. Two numerical examples are designed to test the influence of introducing the proposed pricing strategy as compared to the traditional PP and FP.

The first example illustrates that FP and NP can both attract more users due to the low cost compared to PP. FP will increase the supply and demand gap when bike supply is insufficient. NP can guide the travellers to change their modes or paths to achieve a more balanced bike relocation. It also demonstrates that the proposed dynamic traffic assignment model can capture the dynamic supply-demand interactions in bike sharing system. The second example is conducted to illustrate the operation performance under different pricing strategies. With limited resources in initial promotion stage, NP performs better than FP and PP in various aspects, such as user attraction and fare revenue. While this is the first attempt to provide a theoretical justification of negative pricing scheme in a dockless bike sharing system, many assumptions have been made to ensure the problem to be more simplified and tractable, which at the same time bring limitations to the research outcomes. These assumptions are expected to be relaxed in the future studies to further analyse the optimal negative pricing scheme in the practical operation of dockless bike sharing system.

\section{Acknowledgement}

This research is partly supported by the Singapore Ministry of Education (MOE) AcRF Tier 2 Grant MOE2016-T2-1-044. 


\section{Reference}

Brandstätt, C., Brunekreeft, G., \& Jahnke, K. (2011). How to deal with negative power price spikes? - Flexible voluntary curtailment agreements for large-scale integration of wind. Energy Policy, 39(6), 3732-3740.

Brihaye, T., Geeraerts, G., Krishna, S. N., Manasa, L., Monmege, B., \& Trivedi, A. (2014, September). Adding negative prices to priced timed games. In International Conference on Concurrency Theory (pp. 560-575). Springer, Berlin, Heidelberg.

Caggiani, L., Camporeale, R., Ottomanelli, M., \& Szeto, W. Y. (2018). A modeling framework for the dynamic management of free-floating bike-sharing systems. Transportation Research Part C: Emerging Technologies, 87, 159-182.

CEIBS. (2017). Mobike- dockless bike sharing. Access from http://cn.ceibs.edu/node/10622

Chakirov, A., \& Fourie, P. J. (2014). Enriched sioux falls scenario with dynamic and disaggregate demand. Arbeitsberichte Verkehrs-und Raumplanung, 978.

Chemla, D., Meunier, F., \& Calvo, R. W. (2013a). Bike sharing systems: Solving the static rebalancing problem. Discrete Optimization, 10(2), 120-146.

Chemla, D., Meunier, F., Pradeau, T., Calvo, R. W., \& Yahiaoui, H. (2013b). Selfservice bike sharing systems: simulation, relocation, pricing.

Chen, Y., \& Wang, H. (2018). Pricing for a last-mile transportation system. Transportation Research Part B: Methodological, 107, 57-69.

Ciari, F., Balac, M., \& Balmer, M. (2015). Modelling the effect of different pricing schemes on free-floating carsharing travel demand: a test case for Zurich, Switzerland. Transportation, 42(3), 413-433.

Dantzig, G. B., Eaves, B. C., \& Gale, D. (1979). An algorithm for a piecewise linear model of trade and production with negative prices and bankruptcy. Mathematical Programming, 16(1), 190-209.

Dell'Amico, M., Hadjicostantinou, E., Iori, M., \& Novellani, S. (2014). The bike sharing rebalancing problem: Mathematical formulations and benchmark instances. Omega, 45, 7-19.

DeMaio, P. (2009). Bike-sharing: History, impacts, models of provision, and future. Journal of Public Transportation, 12(4), 41-56.

Dötterl, J., Bruns, R., Dunkel, J., \& Ossowski, S. (2017, September). Towards dynamic rebalancing of bike sharing systems: An event-driven agents approach. In Portuguese Conference on Artificial Intelligence (pp. 309-320). Springer, Cham

Erdoğan, G., Battarra, M., \& Calvo, R. W. (2015). An exact algorithm for the static rebalancing problem arising in bicycle sharing systems. European Journal of Operational Research, 245(3), 667-679.

Forma, I. A., Raviv, T., \& Tzur, M. (2015). A 3-step math heuristic for the static relocation problem in bike-sharing systems. Transportation Research Part B: Methodological, 71, 230-247. 
Fried, I. (2010). Microsoft kills Bing Cashback. CNET. Access from: https://www.cnet.com/news/microsoft-kills-bing-cashback/

Ghosh, S., Varakantham, P., Adulyasak, Y., \& Jaillet, P. (2017). Dynamic repositioning to reduce lost demand in bike sharing systems. Journal of Artificial Intelligence Research, 58, 387-430.

Ho, S. C., \& Szeto, W. Y. (2014). Solving a static relocation problem in bike-sharing systems using iterated tabu search. Transportation Research Part E: Logistics and Transportation Review, 69, 180-198.

Ho, S. C., \& Szeto, W. Y. (2017). A hybrid large neighborhood search for the static multi-vehicle bike-relocation problem. Transportation Research Part B: Methodological, 95, 340-363.

Huang, H. J., \& Lam, W. H. (2002). Modeling and solving the dynamic user equilibrium route and departure time choice problem in network with queues. Transportation Research Part B: Methodological, 36(3), 253-273.

Kadri, A. A., Kacem, I., \& Labadi, K. (2016). A branch-and-bound algorithm for solving the static rebalancing problem in bicycle-sharing systems. Computers \& Industrial Engineering, 95, 41-52.

Laporte, G., Meunier, F., \& Calvo, R. W. (2015). Shared mobility systems. 4or, 13(4), 341-360.

Li, Q., Liao, F., Timmermans, H. J., Huang, H., \& Zhou, J. (2018). Incorporating freefloating car-sharing into an activity-based dynamic user equilibrium model: a demandside model. Transportation Research Part B: Methodological, 107, 102-123.

Liu, W., \& Geroliminis, N. (2017). Doubly dynamics for multi-modal networks with park-and-ride and adaptive pricing. Transportation Research Part B: Methodological, 102, 162-179.

Liu, W., \& Szeto, W. Y. (2019). Learning and managing stochastic network traffic dynamics with an aggregate traffic representation. Transportation Research Part B: Methodological. https://doi.org/10.1016/j.trb.2019.03.021

Lim, A. (2018). Shared-bicycle operators to be licensed to curb indiscriminate parking. Straits Times. Access from: http://www.straitstimes.com/singapore/transport/sharedbicycle-operators-to-be-licensed-to-curb-indiscriminate-parking

Long, J., Szeto, W. Y., Du, J., \& Wong, R. C. P. (2017). A dynamic taxi traffic assignment model: A two-level continuum transportation system approach. Transportation Research Part B: Methodological, 100, 222-254.

Lo, H. K., \& Szeto, W. Y. (2002). A cell-based variational inequality formulation of the dynamic user optimal assignment problem. Transportation Research Part B: Methodological, 36(5), 421-443.

$\mathrm{Lu}$, C. C. (2016). Robust multi-period fleet allocation models for bike-sharing systems. Networks and Spatial Economics, 16(1), 61-82.

Lu, C. C., Mahmassani, H. S., \& Zhou, X. (2008). A bi-criterion dynamic user equilibrium traffic assignment model and solution algorithm for evaluating dynamic 
road pricing strategies. Transportation Research Part C: Emerging Technologies, 16(4), 371-389.

Meng, M., Shao, C. F., Wong, Y. D., \& Zhang, J. (2014). Multimodal network equilibrium with stochastic travel times. Mathematical Problems in Engineering, 2014.

Merchant, D. K., \& Nemhauser, G. L. (1978). A model and an algorithm for the dynamic traffic assignment problems. Transportation science, 12(3), 183-199.

Nagurney, A., \& Zhang, D. (1997). Projected dynamical systems in the formulation, stability analysis, and computation of fixed-demand traffic network equilibria. Transportation Science, 31(2), 147-158.

Nicolosi, M. (2010). Wind power integration and power system flexibility-An empirical analysis of extreme events in Germany under the new negative price regime. Energy Policy, 38(11), 7257-7268.

Pal, A., \& Zhang, Y. (2017). Free-floating bike sharing: solving real-life large-scale static rebalancing problems. Transportation Research Part C: Emerging Technologies, 80, 92-116.

Pfrommer, J., Warrington, J., Schildbach, G., \& Morari, M. (2014). Dynamic vehicle redistribution and online price incentives in shared mobility systems. IEEE Transactions on Intelligent Transportation Systems, 15(4), 1567-1578.

Qian, X., Zhang, W., Ukkusuri, S. V., \& Yang, C. (2017). Optimal assignment and incentive design in the taxi group ride problem. Transportation Research Part B: Methodological, 103, 208-226.

Raviv, T., Tzur, M., \& Forma, I. A. (2013). Static relocation in a bike-sharing system: models and solution approaches. EURO Journal on Transportation and Logistics, 2(3), 187-229.

Regue, R., \& Recker, W. (2014). Proactive vehicle routing with inferred demand to solve the bikesharing rebalancing problem. Transportation Research Part E: Logistics and Transportation Review, 72, 192-209.

Reiss, S., \& Bogenberger, K. (2015, September). GPS-Data analysis of Munich's freefloating bike sharing system and application of an operator-based relocation strategy. In Intelligent Transportation Systems (ITSC), 2015 IEEE 18th International Conference on (pp. 584-589). IEEE.

Reiss, S., \& Bogenberger, K. (2016). Validation of a relocation strategy for Munich's bike sharing system. Transportation Research Procedia, 19, 341-349.

Reiss, S., \& Bogenberger, K. (2017). A relocation strategy for Munich's bike sharing system: combining an operator-based and a user-based scheme. Transportation Research Procedia, 22, 105-114.

Schuijbroek, J., Hampshire, R. C., \& Van Hoeve, W. J. (2017). Inventory rebalancing and vehicle routing in bike sharing systems. European Journal of Operational Research, 257(3), 992-1004.

Simon, H. (2016). Negative prices - A new phenomenon. The Journal of Professional Pricing. Q4, 18-21. 
Shui, C. S., \& Szeto, W. Y. (2018). Dynamic green bike relocation problem-A hybrid rolling horizon artificial bee colony algorithm approach. Transportation Research Part D: Transport and Environment, 60, 119-136.

Singla, A., Santoni, M., Bartók, G., Mukerji, P., Meenen, M., \& Krause, A. (2015, January). Incentivizing users for balancing bike sharing systems. In AAAI (pp. 723729).

Szeto, W. Y., \& Lo, H. K. (2006). Dynamic traffic assignment: properties and extensions. Transportmetrica, 2(1), 31-52.

Szeto, W. Y., Liu, Y., \& Ho, S. C. (2016). Chemical reaction optimization for solving a static bike relocation problem. Transportation Research Part D: Transport And Environment, 47, 104-135.

Szeto, W. Y., \& Shui, C. S. (2018). Exact loading and unloading strategies for the static multi-vehicle bike relocation problem. Transportation Research Part B: Methodological, 109, 176-211.

Tversky, A., \& Kahneman, D. (1992). Advances in prospect theory: Cumulative representation of uncertainty. Journal of Risk and Uncertainty, 5(4), 297-323.

Wang, D. Z., \& Du, B. (2016). Continuum modelling of spatial and dynamic equilibrium in a travel corridor with heterogeneous commuters-A partial differential complementarity system approach. Transportation Research Part B: Methodological, 85, 1-18.

Working, H. (1949). The theory of price of storage. The American Economic Review, 39(6), 1254-1262.

Wright, B. D., \& Williams, J. C. (1989). A theory of negative prices for storage. Journal of Futures Markets, 9(1), 1-13.

Wu, Z., \& Lam, W. (2003). Combined modal split and stochastic assignment model for congested networks with motorized and nonmotorized transport modes. Transportation Research Record: Journal of the Transportation Research Board, (1831), 57-64.

Xu, M., Meng, Q., \& Liu, Z. (2018). Electric vehicle fleet size and trip pricing for oneway carsharing services considering vehicle relocation and personnel assignment. Transportation Research Part B: Methodological, 111, 60-82.

Yan, S., Lin, J. R., Chen, Y. C., \& Xie, F. R. (2017). Rental bike location and allocation under stochastic demands. Computers \& Industrial Engineering, 107, 1-11.

Zhang, F., \& Liu, W. (2019). Responsive bus dispatching strategy in a multi-modal and multi-directional transportation system: A doubly dynamical approach. Transportation Research Part C: Emerging Technologies. https://doi.org/10.1016/j.trc.2019.04.005

Zhang, J., Wang, D. Z., \& Meng, M. (2017). Analyzing customized bus service on a multimodal travel corridor: an analytical modeling approach. Journal of Transportation Engineering, Part A: Systems, 143(11), 04017057.

Zhang, K., Mahmassani, H. S., \& Lu, C. C. (2013). Dynamic pricing, heterogeneous users and perception error: Probit-based bi-criterion dynamic stochastic user 
equilibrium assignment. Transportation Research Part C: Emerging Technologies, 27, 189-204.

Zheng, N., Waraich, R. A., Axhausen, K. W., \& Geroliminis, N. (2012). A dynamic cordon pricing scheme combining the Macroscopic Fundamental Diagram and an agent-based traffic model. Transportation Research Part A: Policy and Practice, 46(8), 1291-1303. 


\section{Appendix}

Proof. We first prove the necessity that the ideal dynamic user equilibrium superpath choice conditions Eqs. (21) - (23) imply variational inequality (43). For any path $p$, a feasible inflow at time $t$ is

$$
f^{w, p, k}(t) \geq 0, \forall w, p, k
$$

Multiplying ideal dynamic user equilibrium super path choice condition Eq. (21) by the (43), it leads to

$$
f^{w, p, k}(t) \cdot\left[P U^{w, p, k *}(t)-\Gamma^{w, p, k *}(t)\right] \geq 0, \forall w, p, k
$$

Subtracting Eq. (22) from Eq. (A2), one can obtain

$$
\left[f^{w, p, k}(t)-f^{w, p, k^{*}}(t)\right] \cdot\left[P U^{w, p, k^{*}}(t)-\Gamma^{w, p, k^{*}}(t)\right] \geq 0, \forall w, p, k
$$

Summing up Eq. (A3) for all paths $p$ and all OD pairs, it follows that

$$
\sum_{w} \sum_{p}\left\{\left[f^{w, p, k}(t)-f^{w, p, k *}(t)\right] \cdot P U^{w, p, k *}(t)\right\} \geq 0, \forall w, p, k
$$

where the flow conversation equation

$$
\sum_{p} f^{w, p, k}(t)=\sum_{p} f^{w, p, k *}(t)=f^{w, k *}(t)
$$

holds for each OD pair at each time interval $t$. Integrating Eq. (A5) from 0 to $\mathrm{T}$, we can obtain variational inequality (43).

Next, we prove the sufficiency that any solution $f^{w, p, k^{*}}(t)$ to (43) satisfies ideal dynamic user equilibrium super path choice conditions Eqs. (21)-(23). We know that the first and third ideal dynamic user equilibrium super path choice conditions Eqs. (21)-(23) hold by definition. Thus, we need to prove that the second ideal dynamic user equilibrium super path choice condition Eq. (22) also holds.

Assume that the second ideal dynamic user equilibrium super path choice condition Eq. (22) does not hold only for a path $p^{\prime}$ for OD pair $w^{\prime}$ during time interval

$$
\begin{aligned}
& {\left[t_{1}-\theta, t_{1}+\theta\right] \in[0, T]} \\
& \quad f^{w^{\prime}, p^{\prime}, k}(t)>0 \text { and } P U^{w^{\prime}, p^{\prime}, k^{*}}(t)-\Gamma^{w^{\prime}, p^{\prime}, k *}(t)>0, \forall t \in\left[t_{1}-\theta, t_{1}+\theta\right]
\end{aligned}
$$

Since the Eq. (22) holds for all paths other than path $p^{\prime}$ for OD pair $w^{\prime}$ at any time interval $t$ and for OD pair $w^{\prime}$ at any time interval $t \notin\left[t_{1}-\theta, t_{1}+\theta\right]$, it follows that

$$
\begin{aligned}
& \sum_{w} \sum_{p} \sum_{t}\left[P U^{w, p, k *}(t)-\Gamma^{w, p, k^{*}}(t)\right] \cdot f^{w, p, k *}(t) \\
= & \sum_{t \in\left[t_{1}-\theta, t_{1}+\theta\right]}\left[P U^{w^{\prime}, p^{\prime}, k^{*}}(t)-\Gamma^{w^{\prime}, p^{\prime}, k^{*}}(t)\right] \cdot f^{w^{\prime}, p^{\prime}, k *}(t)>0
\end{aligned}
$$

Note that all other terms in the above equation vanish because of Eq. (22). For each OD pair $w$, we can always find one minimal actual travel time path $\bar{p}$ for travellers 
departing origin at time $t$, where path $\bar{p}$ was evaluated under the optimal inflow pattern $f^{w, p, k^{*}}(t)$. For this path $\bar{p}$, Eq. (21) becomes an equality by definition. It follows that

$$
P U^{w, \bar{p}, k *}(t)-\Gamma^{w, \bar{p}, k *}(t)=0, \forall w, \bar{p}, k
$$

Next, we need to find a set of feasible path inflows $f^{w, p, k}(t)$ satisfying:

$$
\left[P U^{w, p, k^{*}}(t)-\Gamma^{w, p, k *}(t)\right] \cdot f^{w, p, k}(t)=0, \forall w, p, k
$$

We consider all the departure flows $f^{w, k}(t)$ for all OD pairs at each time interval $t$. For each OD pair $w$ at each time t, we assign OD departure flow $f^{w, k}(t)$ to the minimal travel time path $\bar{p}$, which was evaluated under the optimal flow pattern $f^{w, p, k *}(t)$. This generates a set of feasible path inflow patterns $f^{w, p, k^{*}}(t)$ in which Eq. (A9) is always satisfied because flows are not assigned to paths with non-minimal travel times evaluated under the optimal path inflow pattern $f^{w, p, k^{*}}(t)$. Summing equations (A9) for all paths $p$ and all OD pairs $w$, it follows that

$$
\sum_{w} \sum_{p}\left[P U^{w, p, k^{*}}(t)-\Gamma^{w, p, k^{*}}(t)\right] \cdot f^{w, p, k}(t)=0, \forall w, p, k
$$

Integrating the above equation for time period $[0, \mathrm{~T}]$, we have

$$
\sum_{t} \sum_{w} \sum_{p}\left[P U^{w, p, k *}(t)-\Gamma^{w, p, k^{*}}(t)\right] \cdot f^{w, p, k}(t)=0, \forall w, p, k, t
$$

We subtract Eq. (A6) from Eq. (A11) and obtain

$$
\begin{aligned}
& \sum_{t} \sum_{w} \sum_{p}\left[P U^{w, p, k}(t)-\Gamma^{w, p, k}(t)\right] \cdot\left[f^{w, p, k}(t)-f^{w, p, k^{*}}(t)\right] \\
& =\sum_{t} \sum_{w} \sum_{p} P U^{w, p, k^{*}}(t) \cdot\left[f^{w, p, k}(t)-f^{w, p, k^{*}}(t)\right]<0
\end{aligned}
$$

where the flow conversation equation

$$
\sum_{p} f^{w, p, k}(t)=\sum_{p} f^{w, p, k *}(t)=f^{w, k *}(t)
$$

holds for each OD pair $w$ at each time interval $t$ so that the second term vanishes. The above equation contradicts variational inequality problem (43). Therefore, any optimal solution $f^{w, p, k^{*}}(t)$ to variational inequality (43) satisfies Eq. (22). Since we proved the necessity and sufficiency of the equivalence of variational inequality Eq.(43) to Eqs. (21)-(23), the proof is complete. 